\title{
GEVREY PROPERTIES AND SUMMABILITY OF FORMAL POWER SERIES SOLUTIONS OF SOME INHOMOGENEOUS LINEAR CAUCHY-GOURSAT PROBLEMS
}

\author{
PASCAL REMY
}

\begin{abstract}
In this article, we investigate the Gevrey and summability properties of the formal power series solutions of some inhomogeneous linear CauchyGoursat problems with analytic coefficients in a neighborhood of $(0,0) \in \mathbb{C}^{2}$. In particular, we give necessary and sufficient conditions under which these solutions are convergent or are $k$-summable, for a convenient positive rational number $k$, in a given direction.
\end{abstract}

\section{Contents}

1. Setting the problem

2. Formal series solutions

3. Newton polygon

4. Gevrey order

5. Summability

Appendix A. Gevrey asymptotic

\section{Setting the Problem}

For several years, various works have been done on the divergent solutions of some classes of linear (see $[1,3-6,8,11,17,18,27-34,36,42-45]$ etc.), nonlinear (see $[12-14,19,20,23,24,38]$ etc.) or singular (see $[9,10,21,22,37]$ etc.) partial differential equations or integro-differential equations in two variables or more, allowing thus to formulate many results on Gevrey properties, summability or multisummability.

In this paper, we are interested in the formal power series solutions of linear Cauchy-Goursat problems of the form

$$
\left\{\begin{array}{l}
L U=\widetilde{q}(t, x), \quad L:=\partial_{t}^{\kappa} \partial_{x}^{p}-\sum_{i \in \mathcal{K} q \in Q_{i}} t^{v_{i, q}} a^{(i, q)}(t, x) \partial_{t}^{\kappa-i} \partial_{x}^{q} \\
U(t, x)-w(t, x)=O\left(t^{\kappa} x^{p}\right),
\end{array}\right.
$$

where

- the partial differential operator $L$ satisfies conditions:

$\left(C_{1}\right): \kappa \geqslant 1$ is a positive integer and $p \geqslant 0$ is a nonnegative integer,

2000 Mathematics Subject Classification. 35C10, 35C20, 40B05.

Key words and phrases. Linear partial differential equation, linear integro-differential equation, divergent power series, Newton polygon, Gevrey order, Gevrey asymptotic, summability. 
$\left(C_{2}\right): \mathcal{K}$ is a subset of $\{0, \ldots, \kappa\}$ which contains at least one positive element and which does not contain 0 if $p=0$,

$\left(C_{3}\right): Q_{0}$ is a non-empty subset of $\{0, \ldots, p-1\}$ if $0 \in \mathcal{K}$,

$\left(C_{4}\right): Q_{i}$ is a non-empty finite subset of $\mathbb{N}(=$ the set of nonnegative integers) for all $i \in \mathcal{K}, i \neq 0$,

$\left(C_{5}\right): v_{i, q} \geqslant 0$ is a nonnegative integer and the coefficients $a^{(i, q)}(t, x)$ are holomorphic in the two variables $t$ and $x$ in a polydisc $D_{\rho_{1}} \times D_{\rho_{2}}$ centered at the origin $(0,0) \in \mathbb{C}^{2}\left(D_{\rho_{j}}\right.$ denotes the disc with center 0 and radius $\left.\rho_{j}>0\right)$ for all $i \in \mathcal{K}$ and $q \in Q_{i}$,

$\left(C_{6}\right): a^{(i, q)}(0, x) \not \equiv 0$ for all $i \in \mathcal{K}$ and $q \in Q_{i}$.

- the inhomogeneity $\widetilde{q}(t, x) \in \mathcal{O}\left(D_{\rho_{2}}\right)[[t]]^{1}$ is a formal series in $t$ with coefficients in $\mathcal{O}\left(D_{\rho_{2}}\right)$ which may be smooth, or not,

- the Cauchy-Goursat data $w(t, x)$ is holomorphic in $D_{\rho_{1}} \times D_{\rho_{2}}$.

Under more or less restrictive conditions on valuations $v_{i, q}$, coefficients $a^{(i, q)}(t, x)$, degrees $Q_{i}$, inhomogeneity $\widetilde{q}(t, x)$ and initial data $w(t, x)$, problem (1.1) was already investigated by many authors (see $[1,3-6,8,11,17,18,32-34,36,42-45]$ etc.). Here, we consider the very general problem of the form (1.1), where no generic assumption is made.

For both practical and notational conveniences, we now change the unknown function $U$ to $u$ by

$$
U(t, x)=w(t, x)+\partial_{t}^{-\kappa} \partial_{x}^{-p} u(t, x) .
$$

Then, problem (1.1) is equivalent to the following integro-differential equation

$$
D u=\tilde{f}(t, x), \quad D:=1-\sum_{i \in \mathcal{K}} \sum_{q \in Q_{i}} t^{v_{i, q}} a^{(i, q)}(t, x) \partial_{t}^{-i} \partial_{x}^{q-p}
$$

where the inhomogeneity $\widetilde{f}(t, x) \in \mathcal{O}\left(D_{\rho_{2}}\right)[[t]]$ is defined by

$$
\widetilde{f}(t, x):=\widetilde{q}(t, x)-L w(t, x) .
$$

Notation $\partial_{t}^{-1} u$ stands for the anti-derivative $\int_{0}^{t} u(s, x) d s$ of $u$ with respect to $t$ which vanishes at $t=0$. Recall that the Cauchy formula for repeated integration implies $\partial_{t}^{-\ell} u=\int_{0}^{t} u(s, x) \frac{(t-s)^{\ell-1}}{(\ell-1) !} d s$ for all $\ell \geqslant 1$; hence, in particular, $\partial_{t}^{-\ell}\left(\frac{t^{j}}{j !}\right)=$ $\frac{t^{j+\ell}}{(j+\ell) !}$ for all $\ell \geqslant 1$ and $j \geqslant 0$. It is the same for $\partial_{x}^{-\ell}$ with $\ell \geqslant 1$.

Notation 1.1. For any series $\widetilde{u}(t, x) \in \mathcal{O}\left(D_{\rho_{2}}\right)[[t]]$, we denote in the sequel

$$
\widetilde{u}(t, x)=\sum_{j \geqslant 0} u_{j, *}(x) \frac{t^{j}}{j !}=\sum_{n \geqslant 0} \widetilde{u}_{*, n}(t) \frac{x^{n}}{n !} .
$$

The organization of the paper is as follows. In Section 2, we prove that the linear integro-differential equation (1.2) admits a unique formal series solution $\widetilde{u}(t, x)$ in $\mathcal{O}\left(D_{\rho_{2}}\right)$ [[t]] (Theorem 2.1) and we give a characterization of its coefficients in $\mathcal{O}\left(D_{\rho_{2}}\right)$. In Section 3, we introduce the Newton polygon $N_{t}(D)$ of the operator $D$ at $t=0$ and we give some properties of this. In Section 4 , we show that $\widetilde{u}(t, x)$ and the inhomogeneity $\tilde{f}(t, x)$ are together either convergent or $1 / k$-Gevrey, where $k$ denotes the smallest positive slope of $N_{t}(D)$ (Theorem 4.4). Then, in the latter case, and

\footnotetext{
${ }^{1}$ We denote $\widetilde{q}$ with a tilde to emphasize the possible divergence of the series $\widetilde{q}$.
} 
under four additional conditions on $D$, we investigate the summability of $\widetilde{u}(t, x)$. In particular, we prove in Section 4 (Theorem 5.4) a necessary and sufficient condition under which $\tilde{u}(t, x)$ is $k$-summable in a given direction $\arg (t)=\theta$, generalizing thus the results already obtained by the author in $[42,43]$.

\section{FORMAL SERIES SOLUTIONS}

In this section, we shall be concerned with the formal series solutions in $\mathcal{O}\left(D_{\rho_{2}}\right)[[t]]$ of the linear integro-differential equation (1.2).

Let us first observe that the operator $D$ is a linear operator acting inside $\mathcal{O}\left(D_{\rho_{2}}\right)[[t]]$. Indeed, $\left(\mathcal{O}\left(D_{\rho_{2}}\right)[[t]], \partial_{t}, \partial_{x}\right)$ is a $\mathbb{C}$-differential algebra stable under anti-derivations $\partial_{t}^{-1}$ and $\partial_{x}^{-1}$ and the coefficients $a^{(i, q)}(t, x)$ belong to $\mathcal{O}\left(D_{\rho_{1}} \times D_{\rho_{2}}\right) \subset \mathcal{O}\left(D_{\rho_{2}}\right)[[t]]$ for all $i$ and $q$. More precisely, we have the following.

Theorem 2.1. $D$ is a linear automorphism of $\mathcal{O}\left(D_{\rho_{2}}\right)[[t]]$.

Proof. Let $\tilde{f}(t, x) \in \mathcal{O}\left(D_{\rho_{2}}\right)[[t]]$. Then, a series $\tilde{u}(t, x)=\sum_{j \geqslant 0} u_{j, *}(x) \frac{t^{j}}{j !}$ is solution of $D \widetilde{u}=\tilde{f}(t, x)$ if and only if its coefficients $u_{j, *}(x)$ satisfy, for all $j \geqslant 0$, the identities

$$
\begin{aligned}
& u_{j, *}(x)=f_{j, *}(x)+ \\
& \sum_{i \in \mathcal{K}} \sum_{q \in Q_{i}} \sum_{m=0}^{j-v_{i, q}-i} \frac{j !}{\left(j-v_{i, q}-m\right) !} \frac{a_{m, *}^{(i, q)}(x)}{m !} \partial_{x}^{q-p} u_{j-v_{i, q}-i-m, *}(x)
\end{aligned}
$$

where, as usual, the third sum is 0 as soon as $j<v_{i, q}+i$. Observe that the index $j-v_{i, q}-i-m$ is $<j$ when $\left(i, v_{i, q}, m\right) \neq(0,0,0)$ and is $j$ otherwise. Thereby, some terms $\partial_{x}^{q-p} u_{j, *}(x)$ may occur in the right-hand side of (2.1) and this, only for the $q \in Q_{0}$ satisfying $v_{0, q}=0$. In particular, when terms $\partial_{x}^{q-p} u_{j, *}(x)$ occur, we necessarily have $q-p \in\{-p, \ldots,-1\}$. Then, Lemma 2.2 below proves that equation $D \widetilde{u}=\tilde{f}(t, x)$ admits a unique solution $\tilde{u}(t, x) \in \mathcal{O}\left(D_{\rho_{2}}\right)[[t]]$. Hence, the bijectivity of $D$, which completes the proof.

Lemma 2.2. The linear integro-differential equation

$$
y+\alpha_{1}(x) \partial_{x}^{-1} y+\alpha_{2}(x) \partial_{x}^{-2} y+\ldots+\alpha_{p}(x) \partial_{x}^{-p} y=g(x)
$$

whose coefficients $\alpha_{q}(x)$ and inhomogeneity $g(x)$ are holomorphic in $D_{\rho_{2}}$, posseses exactly one solution $y(x)$. Moreover, this solution is holomorphic in $D_{\rho_{2}}$.

Proof. Let $z=\partial_{x}^{-p} y$. Then, $y(x)$ is a solution of equation (2.2) if and only if $z(x)$ is a solution of the Cauchy problem

$$
\left\{\begin{array}{l}
\partial_{x}^{p} z+\alpha_{1}(x) \partial_{x}^{p-1} z+\alpha_{2}(x) \partial_{x}^{p-2} z+\ldots+\alpha_{p}(x) z=g(x) \\
z(0)=\partial_{x} z(0)=\ldots=\partial_{x}^{p-1} z(0)=0
\end{array}\right.
$$

The result follows then from the Cauchy-Kovalevskaïa theorem for the ordinary differential equations.

As a direct consequence of Theorem 2.1, we deduce in particular that equation (1.2) is uniquely solvable in $\mathcal{O}\left(D_{\rho_{2}}\right)[[t]]$.

Corollary 2.3. The linear integro-differential equation (1.2) admits a unique formal series solution $\tilde{u}(t, x) \in \mathcal{O}\left(D_{\rho_{2}}\right)[[t]]$. Moreover, its coefficients $u_{j, *}(x) \in$ $\mathcal{O}\left(D_{\rho_{2}}\right)$ are recursively determined for all $j \geqslant 0$ by identities (2.1). 
Observe that the formal solution $\widetilde{u}(t, x)$ is divergent in general. In Section 4, we shall investigate its Gevrey properties. We propose in particular to prove a necessary and sufficient condition under which it is $s$-Gevrey with a convenient nonnegative rational number $s$.

Before stating our main result (see Theorem 4.4), let us first introduce the $t$ Newton polygon of the operator $D$.

\section{NEWTON POLYGON}

As definition of the $t$-Newton polygon of the operator $D$ (or Newton polygon of $D$ with respect to $t$ ), we choose the definition of M. Miyake [32] (see also A. Yonemura [45] or S. Ouchi [36]) which is an analogue to the one given by J.-P. Ramis [41] for the linear ordinary differential operators. Recall that H. Tahara and H. Yamazawa use in [44] a slightly different one.

For any $(a, b) \in \mathbb{R}^{2}$, we denote by $C(a, b)$ the domain

$$
C(a, b)=\left\{(x, y) \in \mathbb{R}^{2} ; x \leqslant a \text { and } y \geqslant b\right\} .
$$

Then, the $t$-Newton polygon of $D$ is defined as follows.

Definition 3.1. One calls $t$-Newton polygon of $D$ the convex hull $N_{t}(D)$ of the union of the sets $C(0,0)$ and $C\left(q-p-i, v_{i, q}+i\right)$ for $i \in \mathcal{K}$ and $q \in Q_{i}$ :

$$
N_{t}(D)=C H\left[C(0,0) \cup \bigcup_{\substack{i \in \mathcal{K} \\ q \in Q_{i}}} C\left(q-p-i, v_{i, q}+i\right)\right],
$$

where $C H[\cdot]$ denotes the convex hull of the elements in [.].

The following lemma specifies the geometric structure of $N_{t}(D)$.

Lemma 3.2. Let $\mathcal{S}:=\left\{(i, q) ; i \in \mathcal{K}, q \in Q_{i}\right.$ and $\left.q-p-i>0\right\}$ be.

(1) Suppose $\mathcal{S}=\varnothing$. Then, $N_{t}(D)=C(0,0)$. In particular, $N_{t}(D)$ has no side with a positive slope.

(2) Suppose $\mathcal{S} \neq \varnothing$. Then, $N_{t}(D)$ has (at least) one side with a positive slope. Moreover, its smallest positive slope $k$ is given by

$$
k=\min _{(i, q) \in \mathcal{S}}\left(\frac{v_{i, q}+i}{q-p-i}\right) .
$$

Proof. Point 1 is straightforward from the fact that condition $\mathcal{S}=\varnothing$ implies $C(q-$ $\left.p-i, v_{i, q}+i\right) \subset C(0,0)$ for all $i$ and $q$. As for point 2, it suffices to remark, on one hand, that $C\left(q-p-i, v_{i, q}+i\right) \subset C(0,0)$ for all $(i, q) \notin \mathcal{S}$ and, on the other hand, that the segment with two end points $(0,0)$ and $\left(q-p-i, v_{i, q}+i\right)$ has, for all $(i, q) \in \mathcal{S}$, a positive slope equal to $\left(v_{i, q}+i\right) /(q-p-i)$ (the positivity stems from the fact that $q-p<0$ for all $q \in Q_{0}$; hence, $(i, q) \in \mathcal{S}$ implies $i \geqslant 1$ and then $\left.v_{i, q}+i>0\right)$.

Notation 3.3. When $\mathcal{S} \neq \varnothing$, we choose, and fix once and for all, one of the pairs $(i, q) \in \mathcal{S}$ such that the side of slope $k$ of $N_{t}(D)$ is the segment with end points $(0,0)$ and $\left(q-p-i, v_{i, q}+i\right)$ (see Figure 1 below). In the sequel, we denote this pair by $\left(i^{*}, q^{*}\right)$. 


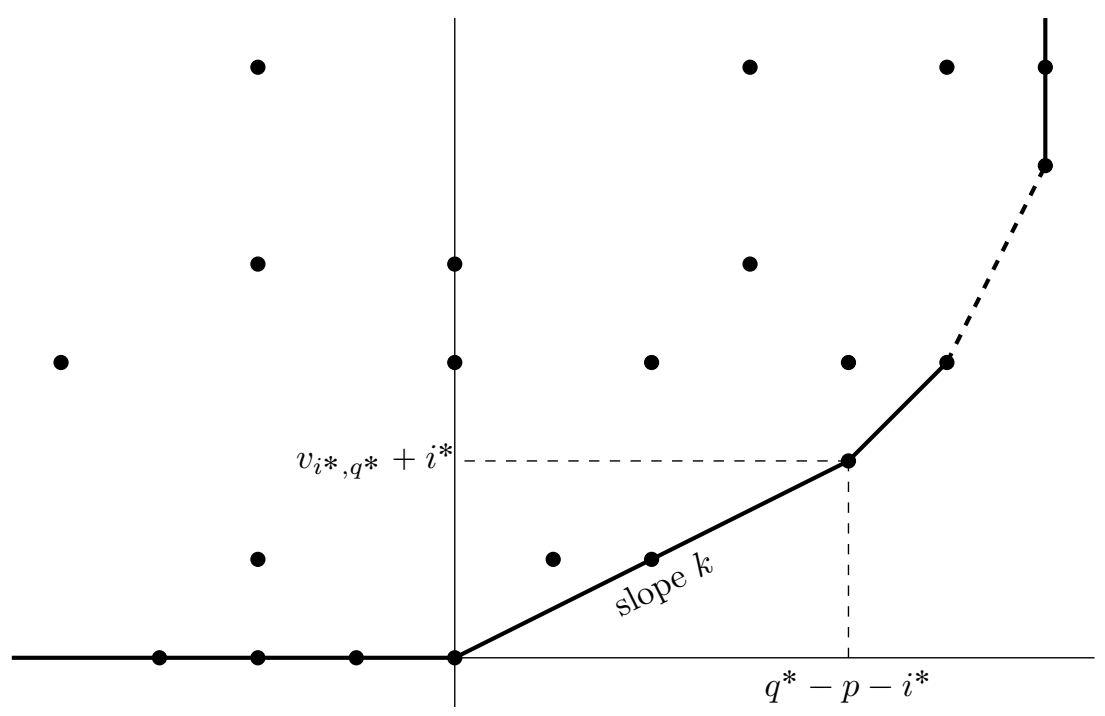

Figure 1. Definition of the pair $\left(i^{*}, q^{*}\right)$.

Remark 3.4. Of course, we have $k=\frac{v_{i^{*}, q^{*}+i^{*}}}{q^{*}-p-i^{*}}$. Moreover, according to the proof of Lemma 3.2, we have besides $q^{*}-p>i^{*} \geqslant 1$.

Let us now turn to the Gevrey properties of $\widetilde{u}(t, x)$.

\section{Gevrey order}

The aim of this section is to investigate the Gevrey properties of the unique formal series $\widetilde{u}(t, x)$ of equation (1.2) (see Corollary 2.3). In particular, we propose to give necessary and sufficient conditions under which it is $s$-Gevrey for some $s \geqslant 0$.

Before stating our main result (see Theorem 4.4 below), let us first recall for the convenience of the reader some definitions and properties about the $s$-Gevrey formal series.

4.1. $s$-Gevrey formal series. All along the article, we consider $t$ as the variable and $x$ as a parameter. Thereby, to define the notion of Gevrey classes of formal power series in $\mathcal{O}\left(D_{\rho_{2}}\right)[[t]]$, one extends the classical notion of Gevrey classes of elements in $\mathbb{C}[[t]]$ to families parametrized by $x$ in requiring similar conditions, the estimates being however uniform with respect to $x$. Doing that, any formal power series of $\mathcal{O}\left(D_{\rho_{2}}\right)[[t]]$ can be seen as a formal power series in $t$ with coefficients in a convenient Banach space defined as the space of functions that are holomorphic on a disc $D_{\rho}\left(0<\rho \leqslant \rho_{2}\right)$ and continuous up to its boundary, equipped with the usual supremum norm. For a general study of series with coefficients in a Banach space, we refer for instance to [2].

Definition 4.1 ( $s$-Gevrey formal series). Let $s \geqslant 0$ be.

A formal series $\widetilde{u}(t, x)=\sum_{j \geqslant 0} u_{j, *}(x) \frac{t^{j}}{j !} \in \mathcal{O}\left(D_{\rho_{2}}\right)[[t]]$ is said to be Gevrey of order $s$ (in short, $s$-Gevrey) if there exist three positive constants $0<r_{2}<\rho_{2}, C>0$ and 
$K>0$ such that the inequalities

$$
\sup _{|x| \leqslant r_{2}}\left|u_{j, *}(x)\right| \leqslant C K^{j} \Gamma(1+(s+1) j)
$$

hold for all $j \geqslant 0$.

In other words, Definition 4.1 means that $\widetilde{u}(t, x)$ is $s$-Gevrey in $t$, uniformly in $x$ on a neighborhood of $x=0$.

We denote by $\mathcal{O}\left(D_{\rho_{2}}\right)[[t]]_{s}$ the set of all the formal series in $\mathcal{O}\left(D_{\rho_{2}}\right)[[t]]$ which are $s$-Gevrey. Observe that the set $\mathbb{C}\{t, x\}$ of germs of analytic functions at the origin $(0,0) \in \mathbb{C}^{2}$ coincides with the union $\bigcup_{\rho>0} \mathcal{O}\left(D_{\rho}\right)[[t]]_{0}$; in particular, any element of $\mathcal{O}\left(D_{\rho_{2}}\right)[[t]]_{0}$ is convergent and $\mathbb{C}\{t, x\} \cap \mathcal{O}\left(D_{\rho_{2}}\right)[[t]]=\mathcal{O}\left(D_{\rho_{2}}\right)[[t]]_{0}$. Observe also that the sets $\mathcal{O}\left(D_{\rho_{2}}\right)[[t]]_{s}$ are filtered as follows:

$$
\mathcal{O}\left(D_{\rho_{2}}\right)[[t]]_{0} \subset \mathcal{O}\left(D_{\rho_{2}}\right)[[t]]_{s} \subset \mathcal{O}\left(D_{\rho_{2}}\right)[[t]]_{s^{\prime}} \subset \mathcal{O}\left(D_{\rho_{2}}\right)[[t]]
$$

for all $s$ and $s^{\prime}$ satisfying $0<s<s^{\prime}<+\infty$.

Following Proposition 4.2 specifies the algebraic structure of the $\mathcal{O}\left(D_{\rho_{2}}\right)[[t]]_{s}$ 's.

Proposition 4.2. Let $s \geqslant 0$ be. Then, $\left(\mathcal{O}\left(D_{\rho_{2}}\right)[[t]]_{s}, \partial_{t}, \partial_{x}\right)$ is a $\mathbb{C}$-differential algebra stable under the anti-derivations $\partial_{t}^{-1}$ and $\partial_{x}^{-1}$.

Proof. See for instance [42, Prop. 1] or [2, p. 64].

4.2. Main result. Let us first begin by observing that Proposition 4.2 implies the following.

Lemma 4.3. $D\left(\mathcal{O}\left(D_{\rho_{2}}\right)[[t]]_{s}\right) \subset \mathcal{O}\left(D_{\rho_{2}}\right)[[t]]_{s}$ for all $s \geqslant 0$.

Theorem 4.4 below specifies this statement by showing more especially that the operator $D$ is actually a linear automorphism of $\mathcal{O}\left(D_{\rho_{2}}\right)[[t]]_{s}$ for some $s \geqslant 0$.

Theorem 4.4. Let $\mathcal{S}:=\left\{(i, q) ; i \in \mathcal{K}, q \in Q_{i}\right.$ and $\left.q-p-i>0\right\}$ and $s$ be the rational number defined by

$$
s:= \begin{cases}0 & \text { if } \mathcal{S}=\varnothing \\ \frac{1}{k}=\frac{q^{*}-p-i^{*}}{v_{i^{*}, q^{*}}+i^{*}} & \text { if } \mathcal{S} \neq \varnothing\end{cases}
$$

Then, $D$ is a linear automorphism of $\mathcal{O}\left(D_{\rho_{2}}\right)[[t]]_{s}$.

In particular, Theorem 4.4 gives us the Gevrey properties of $\widetilde{u}(t, x)$ in view in this section. More precisely, it provides, in the case $\mathcal{S}=\varnothing$, necessary and sufficient condition under which $\widetilde{u}(t, x)$ is convergent and, in the opposite case $\mathcal{S} \neq \varnothing$, necessary and sufficient condition under which $\widetilde{u}(t, x)$ is $s$-Gevrey with $s$ as above.

Corollary 4.5. Let $\mathcal{S}:=\left\{(i, q) ; i \in \mathcal{K}, q \in Q_{i}\right.$ and $\left.q-p-i>0\right\}$ be.

(1) Assume $\mathcal{S}=\varnothing$. Then, $\widetilde{u}(t, x)$ is convergent if and only if the inhomogeneity $\tilde{f}(t, x)$ is convergent.

(2) Assume $\mathcal{S} \neq \varnothing$ and set $s=\frac{q^{*}-p-i^{*}}{v_{i^{*}, q^{*}}+i^{*}}$. Then, $\widetilde{u}(t, x)$ is $s$-Gevrey if and only if the inhomogeneity $\tilde{f}(t, x)$ is s-Gevrey.

As a consequence of Corollary 4.5, we deduce in particular a result similar to the Maillet-Ramis theorem for the ordinary linear differential equations [39,41] (see also [16, Thm. 4.2.7]). 
Corollary 4.6. Assume that the inhomogeneity $\widetilde{f}(t, x)$ is convergent. Then, $\widetilde{u}(t, x)$ is either convergent or s-Gevrey, where $k=1 / s$ is the smallest positive slope of the Newton polygon $N_{t}(D)$ of $D$ with respect to $t$.

4.3. Proof of Theorem 4.4. According to Theorem 2.1 and Lemma 4.3, the operator $D$ is an injective linear operator acting inside $\mathcal{O}\left(D_{\rho_{2}}\right)[[t]]_{s}$. To prove the surjectivity of $D$, we shall use below an approach based on Nagumo norms $[7,35]$ and majorant series; approach which is similar to the ones developed by W. Balser and M. Loday-Richaud in [4] and by the author in $[42,43]$ for some classes of linear integro-differential equations.

4.3.1. Nagumo norms. For the convenience of the reader, we recall in this section the definition of the Nagumo norms and some of their properties which are needed in the sequel.

Definition 4.7 (Nagumo norms). Let $f \in \mathcal{O}\left(D_{\rho}\right), n \geqslant 0$ and $0<r<\rho$ be. Let $d_{r}(x)=r-|x|$ denote the Euclidian distance of $x \in D_{r}$ to the boundary of the disc $D_{r}$. Then, the Nagumo norm $\|f\|_{n, r}$ of $f$ is defined by

$$
\|f\|_{n, r}:=\sup _{|x|<r}\left|f(x) d_{r}(x)^{n}\right| .
$$

Following Proposition 4.8 gives us some properties of the Nagumo norms.

Proposition 4.8 (Properties of Nagumo norms). Let $f, g \in \mathcal{O}\left(D_{\rho}\right), n, n^{\prime} \geqslant 0$ and $0<r<\rho$ be. Then,

(1) $\|\cdot\|_{n, r}$ is a norm on $\mathcal{O}\left(D_{\rho}\right)$.

(2) For all $x \in D_{r},|f(x)| \leqslant\|f\|_{n, r} d_{r}(x)^{-n}$.

(3) $\|f\|_{0, r}=\sup _{|x|<r}|f(x)|$ is the usual sup-norm on $D_{r}$.

(4) $\|f g\|_{n+n^{\prime}, r} \leqslant\|f\|_{n, r}\|g\|_{n^{\prime}, r}$.

(5) $\|\partial f\|_{n+1, r} \leqslant e(n+1)\|f\|_{n, r}$.

(6) $\left\|\partial^{-1} f\right\|_{n, r} \leqslant r\|f\|_{n, r}$.

Proof. Properties 1-4 are straightforward and are left to the reader.

To prove Property 5, we proceed as follows. Let $x \in D_{r}$ and $0<R<d_{r}(x)$ be. Using the Cauchy integral formula, we have

$$
|\partial f(x)|=\frac{1}{2 \pi}\left|\int_{\left|x^{\prime}-x\right|=R} \frac{f\left(x^{\prime}\right)}{\left(x^{\prime}-x\right)^{2}} d x^{\prime}\right| \leqslant \frac{1}{R} \max _{\left|x^{\prime}-x\right|=R}\left|f\left(x^{\prime}\right)\right|
$$

and then

$$
|\partial f(x)| \leqslant\|f\|_{n, r} \frac{1}{R} \max _{\left|x^{\prime}-x\right|=R} d_{r}\left(x^{\prime}\right)^{-n}=\|f\|_{n, r} \frac{1}{R}\left(d_{r}(x)-R\right)^{-n}
$$

by applying Property 2 . Let us now assume $n>0$ and let us choose

$$
R=\frac{d_{r}(x)}{n+1} .
$$

Then, using the inequality $\left(1-\frac{1}{n+1}\right)^{-n}=\left(1+\frac{1}{n}\right)^{n}<e$, we get

$$
|\partial f(x)| \leqslant\|f\|_{n, r} d_{r}(x)^{-n-1}(n+1)\left(1-\frac{1}{n+1}\right)^{-n} \leqslant(n+1) e\|f\|_{n, r} d_{r}(x)^{-n-1}
$$


hence, the result:

$$
\|\partial f\|_{n+1, r}=\sup _{|x|<r}\left|\partial f(x) d_{r}(x)^{n+1}\right| \leqslant(n+1) e\|f\|_{n, r} .
$$

For $n=0$, we set

$$
R=\frac{d_{r}(x)}{c}
$$

with an arbitrary constant $c>1$; hence, the inequality

$$
|\partial f(x)| \leqslant c\|f\|_{0, r} d_{r}(x)^{-1}
$$

and then

$$
\|\partial f\|_{1, r}=\sup _{|x|<r}\left|\partial f(x) d_{r}(x)\right| \leqslant c\|f\|_{0, r} .
$$

The result follows by choosing $c=e$.

We are left to prove Property 6 . Let $x \in D_{r}$ be. Using Property 2, we obtain

$$
\left|\partial^{-1} f(x)\right|=\left|\int_{0}^{x} f(t) d t\right| \leqslant\|f\|_{n, r} \int_{0}^{|x|} \frac{d u}{(r-u)^{n}}
$$

for all $n \geqslant 0$ and, consequently, the following discussion.

- Case $n=0$. Due to inequality (4.1) above, we straightaway have

and then

$$
\left|\partial^{-1} f(x)\right| \leqslant\|f\|_{0, r} \int_{0}^{|x|} d u=|x|\|f\|_{0, r} \leqslant r\|f\|_{0, r}
$$

$$
\left\|\partial^{-1} f\right\|_{0, r}=\sup _{|x|<r}\left|\partial^{-1} f(x)\right| \leqslant r\|f\|_{0, r} .
$$

- Case $n=1$. Using inequalities (4.1) and $\ln (t) \leqslant t$ for all $t>0$, we have

$$
\left|\partial^{-1} f(x)\right| \leqslant\|f\|_{1, r} \int_{0}^{|x|} \frac{d u}{r-u}=\|f\|_{1, r} \ln \left(\frac{r}{d_{r}(x)}\right) \leqslant \frac{r}{d_{r}(x)}\|f\|_{1, r} .
$$

Hence, the result:

$$
\left\|\partial^{-1} f\right\|_{1, r}=\sup _{|x|<r}\left|\partial^{-1} f(x) d_{r}(x)\right| \leqslant r\|f\|_{1, r} .
$$

- Case $n \geqslant 2$. Since

$$
\int_{0}^{|x|} \frac{d u}{(r-u)^{n}}=\frac{1}{(n-1) d_{r}(x)^{n-1}}-\frac{1}{r(n-1)} \leqslant \frac{1}{d_{r}(x)^{n-1}}
$$

inequality (4.1) implies

$$
\left|\partial^{-1} f(x) d_{r}(x)^{n}\right| \leqslant\|f\|_{n, r} d_{r}(x) \leqslant r\|f\|_{n, r} .
$$

Hence, the result again:

$$
\left\|\partial^{-1} f\right\|_{n, r}=\sup _{|x|<r}\left|\partial^{-1} f(x) d_{r}(x)^{n}\right| \leqslant r\|f\|_{n, r} .
$$

This achieves the proof of Proposition 4.8.

Remark 4.9. Inequalities $4-6$ are the most important properties. Observe that the same index $r$ occurs on their both sides, allowing thus to get estimates for the product $f g$ in terms of $f$ and $g$, for the derivative $\partial f$ in terms of $f$ and for the anti-derivative $\partial^{-1} f$ in terms of $f$ without having to shrink the disc $D_{r}$.

Let us now turn to the proof of Theorem 4.4.

\subsubsection{Proof of Theorem 4.4.}


$\triangleleft$ First step: a fundamental technical lemma.

Before starting the calculations, let us first begin with the following technical lemma which will play a central role in our proof.

Lemma 4.10. Assume $\mathcal{S} \neq \varnothing$. Then, the inequalities

$$
(s+1)\left(v_{i, q}+i\right) \geqslant q-p+v_{i, q}
$$

hold for all $i \in \mathcal{K}$ and $q \in Q_{i}$.

Proof. Inequalities (4.2) are clear when $i=v_{i, q}=0$ (indeed, $q-p<0$ for all $\left.q \in Q_{0}\right)$. When $\left(i, v_{i, q}\right) \neq(0,0)$, we have $v_{i, q}+i \geqslant 1$ and the inequality

$$
s=\frac{1}{k} \geqslant \frac{q-p-i}{v_{i, q}+i}
$$

which stems, on one hand, from the definition of $k$ when $(i, q) \in \mathcal{S}$ and, on the other hand, from the fact that $q-p-i \leqslant 0$ when $(i, q) \notin \mathcal{S}$. Lemma 4.10 follows then by adding " +1 " to both sides of (4.3).

Remark 4.11. In fact, inequalities (4.2) still hold when $\mathcal{S}=\varnothing$. Indeed, we have $s=0$ and $q-p \leqslant i$ for all $i \in \mathcal{K}$ and $q \in Q_{i}$. Nevertheless, we shall only use subsequently these inequalities in the case where $\mathcal{S} \neq \varnothing$. Hence, the statement of Lemma 4.10 as it is written.

We are now able to prove Theorem 4.4.

$\triangleleft$ Second step: preliminaries.

As we said at the beginning of Section 4.3, we are left to prove the surjectivity of the linear integro-differential operator $D$. To do that, let us fix

$$
\tilde{f}(t, x)=\sum_{j \geqslant 0} f_{j, *}(x) \frac{t^{j}}{j !} \in \mathcal{O}\left(D_{\rho_{2}}\right)[[t]]_{s}
$$

and let us write the solution $\widetilde{u}(t, x) \in \mathcal{O}\left(D_{\rho_{2}}\right)[[t]]$ of equation (1.2) in the same form:

$$
\widetilde{u}(t, x)=\sum_{j \geqslant 0} u_{j, *}(x) \frac{t^{j}}{j !} .
$$

By assumption, the coefficients $f_{j, *}(x)$ satisfy the following two conditions

- $f_{j, *}(x) \in \mathcal{O}\left(D_{\rho_{2}}\right)$ for all $j \geqslant 0$,

- there exist three positive constants $0<r_{2}<\rho_{2}, C>0$ and $K>0$ such that $\left|f_{j, *}(x)\right| \leqslant C K^{j} \Gamma(1+(s+1) j)$ for all $j \geqslant 0$ and $|x| \leqslant r_{2}$.

We shall now prove that the coefficients $u_{j, *}(x)$ satisfy similar conditions. The calculations below are analogous to those detailed in $[4,42,43]$, but are much more complicated because of the terms $\partial_{t}^{-i} \partial_{x}^{q-p}$ with $q-p \in \mathbb{Z}$. 
$\triangleleft$ Third step: some inequalities.

From identities (2.1), we obtain the relations

$$
\begin{aligned}
\frac{u_{j, *}(x)}{\Gamma(1+(s+1) j)}= & \frac{f_{j, *}(x)}{\Gamma(1+(s+1) j)}+ \\
& \sum_{i \in \mathcal{K}} \sum_{q \in Q_{i}} \sum_{m=0}^{j-v_{i, q}-i} \frac{j !}{\left(j-v_{i, q}-m\right) !} \frac{a_{m, *}^{(i, q)}(x)}{m !} \frac{\partial_{x}^{q-p} u_{j-v_{i, q}-i-m, *}(x)}{\Gamma(1+(s+1) j)}
\end{aligned}
$$

for all $j \geqslant 0$ (as before, we use the classical convention that the third sum is 0 if $\left.j<v_{i, q}+i\right)$.

Notation 4.12. In the sequel, we denote by $\sigma$ the positive integer ${ }^{2}$ defined by

$$
\sigma:= \begin{cases}v+\kappa & \text { if } \mathcal{S}=\varnothing \\ (s+1)\left(v_{i *, q^{*}}+i^{*}\right) & \text { if } \mathcal{S} \neq \varnothing\end{cases}
$$

where $v$ is the nonnegative integer $v:=\max \left\{v_{i, q} ; i \in \mathcal{K}\right.$ and $\left.q \in Q_{i}\right\}$.

Let us now apply the Nagumo norm of indices $\left(\sigma j, r_{2}\right)$. From Property 4 of Proposition 4.8, we first obtain

$$
\frac{\left\|u_{j, *}(x)\right\|_{\sigma j, r_{2}}}{\Gamma(1+(s+1) j)} \leqslant \frac{\left\|f_{j, *}(x)\right\|_{\sigma j, r_{2}}}{\Gamma(1+(s+1) j)}+\sum_{i \in \mathcal{K}} \sum_{q \in Q_{i}} \sum_{m=0}^{j-v_{i, q}-i} A_{j, i, q, m}(x)
$$

with

$$
A_{j, i, q, m}(x):=\frac{j !}{\left(j-v_{i, q}-m\right) !} \frac{\left\|a_{m, *}^{(i, q)}(x)\right\|_{\sigma\left(v_{i, q}+i+m\right)-\delta_{q}, r_{2}}}{m !} \times
$$

where $\delta_{q}$ is the nonnegative integer defined by

$$
\delta_{q}:= \begin{cases}0 & \text { if } q-p \leqslant 0 \\ q-p & \text { if } q-p>0\end{cases}
$$

Then, Properties 5-6 of Proposition 4.8 imply the inequality

$$
\frac{\left\|u_{j, *}(x)\right\|_{\sigma j, r_{2}}}{\Gamma(1+(s+1) j)} \leqslant \frac{\left\|f_{j, *}(x)\right\|_{\sigma j, r_{2}}}{\Gamma(1+(s+1) j)}+\sum_{i \in \mathcal{K}} \sum_{q \in Q_{i}} \sum_{m=0}^{j-v_{i, q}-i} B_{j, i, q, m}(x)
$$

with

$$
B_{j, i, q, m}(x):=\beta_{j, i, q, m} \frac{\left\|a_{m, *}^{(i, q)}(x)\right\|_{\sigma\left(v_{i, q}+i+m\right)-\delta_{q}, r_{2}}}{m !}\left\|u_{j-v_{i, q}-i-m, *}(x)\right\|_{\sigma\left(j-v_{i, q}-i-m\right), r_{2}},
$$

${ }^{2}$ See Remark 3.4 . 
where $\beta_{j, i, q, m}$ is the nonnegative integer defined by

$$
\beta_{j, i, q, m}:= \begin{cases}\frac{j ! r_{2}^{p-q}}{\left(j-v_{i, q}-m\right) ! \Gamma(1+(s+1) j)} & \text { if } q-p \leqslant 0, \\ \frac{j !\left(\prod_{\ell=0}^{q-p-1}\left(\sigma\left(j-v_{i, q}-i-m\right)+q-p-\ell\right)\right) e^{q-p}}{\left(j-v_{i, q}-m\right) ! \Gamma(1+(s+1) j)} & \text { if } q-p>0 .\end{cases}
$$

Remark 4.13. Norms $\left\|a_{m, *}^{(i, q)}(x)\right\|_{\sigma\left(v_{i, q}+i+m\right), r_{2}}$ and $\left\|u_{j-v_{i, q}-i-m, *}(x)\right\|_{\sigma\left(j-v_{i, q}-i-m\right), r_{2}}$ are both clearly well-defined. Norms $\left\|a_{m, *}^{(i, q)}(x)\right\|_{\sigma\left(v_{i, q}+i+m\right)-(q-p), r_{2}}$ are well-defined too when $q-p>0$. Indeed, in the case $\mathcal{S}=\varnothing$, conditions $\kappa \geqslant 1, i \geqslant 0, v_{i, q} \geqslant 0$ and $q-p \leqslant i$ imply

$$
\sigma\left(v_{i, q}+i+m\right)-(q-p) \geqslant \sigma i-(q-p) \geqslant \kappa i-(q-p) \geqslant \kappa i-i=i(\kappa-1) \geqslant 0
$$

and, in the opposite case $\mathcal{S} \neq \varnothing$, Lemma 4.10 and conditions $i^{*} \geqslant 1$ (see Remark 3.4) and $v_{i, q} \geqslant 0$ imply

$$
\begin{aligned}
\sigma\left(v_{i, q}+i+m\right)-(q-p) & \geqslant \sigma\left(v_{i, q}+i\right)-(q-p) \\
& =(s+1)\left(v_{i^{*}, q^{*}}+i^{*}\right)\left(v_{i, q}+i\right)-(q-p) \\
& \geqslant\left(q-p+v_{i, q}\right)\left(v_{i^{*}, q^{*}}+i^{*}\right)-(q-p) \\
& =(q-p)\left(v_{i^{*}, q^{*}}+i^{*}-1\right)+v_{i, q}\left(v_{i *, q^{*}}+i^{*}\right) \\
& \geqslant 0
\end{aligned}
$$

Following proposition allows us to bound the $\beta_{j, i, q, m}$ 's.

Proposition 4.14. Let $i \in \mathcal{K}, q \in Q_{i}, j \geqslant v_{i, q}+i$ and $m \in\left\{0, \ldots, j-v_{i, q}-i\right\}$ be. Then,

$$
\frac{j !}{\left(j-v_{i, q}-m\right) ! \Gamma(1+(s+1) j)} \leqslant \frac{1}{\Gamma\left(1+(s+1)\left(j-v_{i, q}-i-m\right)\right)} .
$$

Moreover, if $q-p>0$, we have

$$
\frac{j !\left(\prod_{\ell=0}^{q-p-1}\left(\sigma\left(j-v_{i, q}-i-m\right)+q-p-\ell\right)\right)}{\left(j-v_{i, q}-m\right) ! \Gamma(1+(s+1) j)} \leqslant \frac{(v+\kappa)^{q-p}}{\Gamma\left(1+(s+1)\left(j-v_{i, q}-i-m\right)\right)} .
$$

Proof. The first inequality stems from Lemma 4.16 (inequality (4.5)) and Lemma 4.17 and the second one from Lemma 4.16 (inequality (4.6)) and Lemma 4.18.

Remark 4.15. Observe that the second inequality of Proposition 4.14 may occur only when $i \geqslant 1$. Indeed, we have $q<p$ for all $q \in Q_{0}$ (see Condition $\left(C_{3}\right)$ ).

Lemma 4.16. Let $i \in \mathcal{K}, q \in Q_{i}, j \geqslant v_{i, q}+i$ and $m \in\left\{0, \ldots, j-v_{i, q}-i\right\}$ be. Then,

$$
\frac{j !}{\left(j-v_{i, q}-m\right) !} \frac{1}{\Gamma(1+(s+1) j)} \leqslant \frac{1}{\Gamma\left(1+(s+1)\left(j-v_{i, q}-m\right)\right)} .
$$

Moreover, if $i \geqslant 1$, we also have

$$
\frac{j !}{\left(j-v_{i, q}-m\right) !} \frac{1}{\Gamma(1+(s+1) j)} \leqslant \frac{1}{\Gamma\left(1+(s+1)(j-m)-v_{i, q}\right)} .
$$


Proof. Lemma 4.16 is clear when $v_{i, q}+m=0$. Let us now suppose $v_{i, q}+m \geqslant 1$.

$\star$ Proof of inequality (4.5). For $(i, m) \neq\left(0, j-v_{0, q}\right)$, let us write the two factors of the left-hand side of inequality (4.5) as follows:

$$
\begin{aligned}
\frac{j !}{\left(j-v_{i, q}-m\right) !} & =\prod_{\ell=0}^{v_{i, q}+m-1}(j-\ell), \\
\Gamma(1+(s+1) j) & =\Gamma\left(1+(s+1) j-v_{i, q}-m\right) \prod_{\ell=0}^{v_{i, q}+m-1}((s+1) j-\ell) .
\end{aligned}
$$

Then,

$$
\begin{aligned}
\frac{j !}{\left(j-v_{i, q}-m\right) !} \frac{1}{\Gamma(1+(s+1) j)} & =\frac{\prod_{\ell=0}^{v_{i, q}+m-1} \frac{j-\ell}{(s+1) j-\ell}}{\Gamma\left(1+(s+1) j-v_{i, q}-m\right)} \\
& \leqslant \frac{1}{\Gamma\left(1+(s+1) j-v_{i, q}-m\right)} .
\end{aligned}
$$

Observe that these relations make sense since the following inequalities

$$
(s+1) j-\ell \geqslant 1+(s+1) j-v_{i, q}-m \geqslant 1+s j+i \geqslant 1
$$

hold for all $\ell \in\left\{0, \ldots, v_{i, q}+m-1\right\}$. Inequality (4.5) follows then from the increase of the Gamma function on $[2,+\infty[$. Indeed, we have the inequalities

$$
1+(s+1) j-v_{i, q}-m \geqslant 1+(s+1)\left(j-v_{i, q}-m\right) \geqslant 1+(s+1) i \geqslant 2
$$

for $i \geqslant 1$ and the inequalities

$$
1+(s+1) j-v_{0, q}-m \geqslant 1+(s+1)\left(j-v_{0, q}-m\right) \geqslant 2+s \geqslant 2
$$

for $i=0$ and $m \in\left\{0, \ldots, j-v_{0, q}-1\right\}$.

We are left to prove inequality $(4.5)$ for $(i, m)=\left(0, j-v_{0, q}\right)$, that is the inequality

$$
\frac{j !}{\Gamma(1+(s+1) j)}=\frac{\Gamma(1+j)}{\Gamma(1+(s+1) j)} \leqslant 1 \text {. }
$$

This latter is clear for $j=0$ and stems from the inequalities $1+(s+1) j \geqslant 1+j \geqslant 2$ and from the increase of the Gamma function on $[2,+\infty[$ for $j \geqslant 1$.

$\star$ Proof of inequality (4.6). Let $i \geqslant 1$ be. From calculations above, we have

$$
\frac{j !}{\left(j-v_{i, q}-m\right) !} \frac{1}{\Gamma(1+(s+1) j)} \leqslant \frac{1}{\Gamma\left(1+(s+1) j-v_{i, q}-m\right)} .
$$

Then, inequality (4.6) stems as previously from the increase of the Gamma function on $[2,+\infty[$ applied to the inequalities

$$
1+(s+1) j-v_{i, q}-m \geqslant 1+(s+1)(j-m)-v_{i, q} \geqslant 1+(s+1) i+s v_{i, q} \geqslant 2 .
$$

This ends the proof of Lemma 4.16 .

Lemma 4.17. Let $i \in \mathcal{K}, q \in Q_{i}, j \geqslant v_{i, q}+i$ and $m \in\left\{0, \ldots, j-v_{i, q}-i\right\}$ be. Then,

$$
\frac{1}{\Gamma\left(1+(s+1)\left(j-v_{i, q}-m\right)\right)} \leqslant \frac{1}{\Gamma\left(1+(s+1)\left(j-v_{i, q}-i-m\right)\right)} .
$$


Proof. For $m \leqslant j-v_{i, q}-i-1$, we have

$$
1+(s+1)\left(j-v_{i, q}-m\right) \geqslant 1+(s+1)\left(j-v_{i, q}-i-m\right) \geqslant 2+s \geqslant 2
$$

and Lemma 4.17 follows from the increase of the Gamma function on [2, $+\infty$ [. For $m=j-v_{i, q}-i$, we must prove the inequality

$$
\frac{1}{\Gamma(1+(s+1) i)} \leqslant 1
$$

This latter is clear for $i=0$ and stems again from the increase of the Gamma function on $[2,+\infty[$ for $i \geqslant 1$. Indeed, we have the inequalities $1+(s+1) i \geqslant 2+s \geqslant 2$; hence, $\Gamma(1+(s+1) i) \geqslant \Gamma(2)=1$. This achieves the proof.

Lemma 4.18. Let $i \in \mathcal{K}, i \neq 0^{3}, q \in Q_{i}, j \geqslant v_{i, q}+i$ and $m \in\left\{0, \ldots, j-v_{i, q}-i\right\}$ be. Assume $q-p>0$. Then,

$$
\frac{\prod_{\ell=0}^{q-p-1}\left(\sigma\left(j-v_{i, q}-i-m\right)+q-p-\ell\right)}{\Gamma\left(1+(s+1)(j-m)-v_{i, q}\right)} \leqslant \frac{(v+\kappa)^{q-p}}{\Gamma\left(1+(s+1)\left(j-v_{i, q}-i-m\right)\right)} .
$$

Proof. $\star$ Let us first assume $\mathcal{S}=\varnothing$ (hence, $\sigma=v+\kappa$ and $s=0$ ). From the relations $0<q-p \leqslant i \leqslant \kappa \leqslant v+\kappa$ and from the identities

$$
\begin{aligned}
& \prod_{\ell=0}^{q-p-1}\left(\sigma\left(j-v_{i, q}-i-m\right)+q-p-\ell\right)= \\
& \quad(v+\kappa)^{q-p} \prod_{\ell=0}^{q-p-1}\left(j-v_{i, q}-i-m+\frac{q-p-\ell}{v+\kappa}\right)
\end{aligned}
$$

and

$$
\begin{aligned}
\Gamma\left(1+(s+1)(j-m)-v_{i, q}\right) & =\Gamma\left(1+j-m-v_{i, q}\right) \\
& =\Gamma\left(1+j-v_{i, q}-i-m\right) \prod_{\ell=0}^{i-1}\left(j-v_{i, q}-m-\ell\right)
\end{aligned}
$$

we deduce the inequality

$$
\begin{aligned}
\frac{\prod_{\ell=0}^{q-p-1}\left(\sigma\left(j-v_{i, q}-i-m\right)+q-p-\ell\right)}{\Gamma\left(1+(s+1)(j-m)-v_{i, q}\right)} \leqslant & \frac{(v+\kappa)^{q-p}}{\Gamma\left(1+j-v_{i, q}-i-m\right)} \\
& \times \frac{\prod_{\ell=0}^{q-p-1} \frac{j-v_{i, q}-i-m+\frac{q-p-\ell}{v+\kappa}}{j-v_{i, q}-m-\ell}}{\prod_{\ell=q-p}^{i-1}\left(j-v_{i, q}-m-\ell\right)}
\end{aligned}
$$

with the convention that the product $\prod_{\ell=q-p}^{i-1}\left(j-v_{i, q}-m-\ell\right)$ is 1 when $q-p=i$. Observe that $j-v_{i, q}-m-\ell \geqslant 1$ for all $\ell$. Indeed, we have $m \leqslant j-v_{i, q}-i$ and

${ }^{3}$ See Remark 4.15 . 
$\ell \leqslant i-1$. In particular, we obtain

$$
\prod_{\ell=q-p}^{i-1}\left(j-v_{i, q}-m-\ell\right) \geqslant 1
$$

On the other hand, inequalities $0 \leqslant \ell \leqslant q-p-1 \leqslant i-1$ and $q-p \leqslant v+\kappa$ imply

$$
\begin{aligned}
\left(j-v_{i, q}-i-m+\frac{q-p-\ell}{v+\kappa}\right)-\left(j-v_{i, q}-m-\ell\right) & =-i+\frac{q-p-\ell}{v+\kappa}+\ell \\
& \leqslant-i+\frac{q-p}{v+\kappa}+i-1 \\
& \leqslant 0 .
\end{aligned}
$$

Thereby, the following inequality

$$
\prod_{\ell=0}^{q-p-1} \frac{j-v_{i, q}-i-m+\frac{q-p-\ell}{v+\kappa}}{j-v_{i, q}-m-\ell} \leqslant 1
$$

holds; hence,

$$
\begin{aligned}
\frac{\prod_{\ell=0}^{q-p-1}\left(\sigma\left(j-v_{i, q}-i-m\right)+q-p-\ell\right)}{\Gamma\left(1+(s+1)(j-m)-v_{i, q}\right)} & \leqslant \frac{(v+\kappa)^{q-p}}{\Gamma\left(1+j-v_{i, q}-i-m\right)} \\
& =\frac{(v+\kappa)^{q-p}}{\Gamma\left(1+(s+1)\left(j-v_{i, q}-i-m\right)\right)},
\end{aligned}
$$

which proves Lemma 4.18 for $\mathcal{S}=\varnothing$.

$\star$ Let us now assume $\mathcal{S} \neq \varnothing$. Thanks to the relation $s+1=\frac{\sigma}{v_{i^{*}, q^{*}}+i^{*}} \geqslant \frac{\sigma}{v+\kappa}$, we have the following inequality:

$$
\begin{aligned}
& \prod_{\ell=0}^{q-p-1}\left(\sigma\left(j-v_{i, q}-i-m\right)+q-p-\ell\right) \leqslant \\
& \quad(v+\kappa)^{q-p} \prod_{\ell=0}^{q-p-1}\left((s+1)\left(j-v_{i, q}-i-m\right)+\frac{q-p-\ell}{v+\kappa}\right) .
\end{aligned}
$$

Let us now write $\Gamma\left(1+(s+1)(j-m)-v_{i, q}\right)$ in the form

$$
\begin{aligned}
\Gamma\left(1+(s+1)(j-m)-v_{i, q}\right)=\Gamma(1+ & \left.(s+1)(j-m)-v_{i, q}-(q-p)\right) \\
& \times \prod_{\ell=0}^{q-p-1}\left((s+1)(j-m)-v_{i, q}-\ell\right) .
\end{aligned}
$$

Observe that the term $\Gamma\left(1+(s+1)(j-m)-v_{i, q}-(q-p)\right)$ is already well-defined. Indeed, condition $m \leqslant j-v_{i, q}-i$ and Lemma 4.10 imply

$$
1+(s+1)(j-m)-v_{i, q}-(q-p) \geqslant 1+(s+1)\left(v_{i, q}+i\right)-\left(q-p+v_{i, q}\right) \geqslant 1 .
$$


From relations (4.7) and (4.8), we obtain

$$
\begin{array}{r}
\frac{\prod_{\ell=0}^{q-p-1}\left(\sigma\left(j-v_{i, q}-i-m\right)+q-p-\ell\right)}{\Gamma\left(1+(s+1)(j-m)-v_{i, q}\right)} \\
\times \frac{(v+\kappa)^{q-p}}{\Gamma\left(1+(s+1)(j-m)-v_{i, q}-(q-p)\right)} \\
\times \prod_{\ell=0}^{q-p-1} \frac{(s+1)\left(j-v_{i, q}-i-m\right)+\frac{q-p-\ell}{v+\kappa}}{(s+1)(j-m)-v_{i, q}-\ell}
\end{array}
$$

where the product on the right-hand side is $\leqslant 1$. Indeed, Lemma 4.10 and the conditions $\ell<q-p$ and $v+\kappa \geqslant 1$ imply relations

$$
\begin{aligned}
\left((s+1)\left(j-v_{i, q}-i-m\right)+\frac{q-p-\ell}{v+\kappa}\right) & -\left((s+1)(j-m)-v_{i, q}-\ell\right) \\
& =-(s+1)\left(v_{i, q}+i\right)+\frac{q-p-\ell}{v+\kappa}+v_{i, q}+\ell \\
& \leqslant-\left(q-p+v_{i, q}\right)+\frac{q-p-\ell}{v+\kappa}+v_{i, q}+\ell \\
& =(q-p-\ell)\left(\frac{1}{v+\kappa}-1\right) \\
& \leqslant 0
\end{aligned}
$$

Let us now assume $m<j-v_{i, q}-i$. Then, Lemma 4.18 follows from inequalities

$$
\begin{aligned}
1+(s+1)(j-m)-v_{i, q}-(q-p) & =1+(s+1)(j-m)-\left(q-p+v_{i, q}\right) \\
& \geqslant 1+(s+1)(j-m)-(s+1)\left(v_{i, q}+i\right) \\
& =1+(s+1)\left(j-v_{i, q}-i-m\right) \\
& \geqslant 2+s \\
& \geqslant 2
\end{aligned}
$$

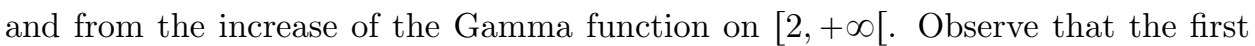
inequality stems from Lemma 4.10 and that the second inequality stems from the condition $m<j-v_{i, q}-i$. In particular, this latter inequality shows that the calculations above do not allow to prove Lemma 4.18 when $m=j-v_{i, q}-i$, since it fails in this case.

To get around this problem, we shall proceed as follows. Let us first recall we must prove the inequality

$$
\frac{\prod_{\ell=0}^{q-p-1}(q-p-\ell)}{\Gamma\left(1+(s+1)\left(v_{i, q}+i\right)-v_{i, q}\right)} \leqslant \frac{(v+\kappa)^{q-p}}{\Gamma(1)}=(v+\kappa)^{q-p} .
$$

From Lemma 4.10 and the condition $q-p>0$, we obtain

$$
1+(s+1)\left(v_{i, q}+i\right)-v_{i, q} \geqslant 1+q-p \geqslant 2
$$

hence, applying the increase of the Gamma function on $[2,+\infty[$, the relation

$$
\Gamma\left(1+(s+1)\left(v_{i, q}+i\right)-v_{i, q}\right) \geqslant \Gamma(1+q-p)=\prod_{\ell=0}^{q-p-1}(q-p-\ell)
$$


and, consequently, the following inequality

$$
\frac{\prod_{\ell=0}^{q-p-1}(q-p-\ell)}{\Gamma\left(1+(s+1)\left(v_{i, q}+i\right)-v_{i, q}\right)} \leqslant 1 .
$$

This achieves the proof since $v+\kappa \geqslant 1$.

Let us now apply Proposition 4.14. We get

$$
\beta_{j, i, q, m}:= \begin{cases}\frac{r_{2}^{p-q}}{\Gamma\left(1+(s+1)\left(j-v_{i, q}-i-m\right)\right)} & \text { if } q-p \leqslant 0, \\ \frac{(e(v+\kappa))^{q-p}}{\Gamma\left(1+(s+1)\left(j-v_{i, q}-i-m\right)\right)} & \text { if } q-p>0 .\end{cases}
$$

Then, the following inequalities

$$
\begin{aligned}
\frac{\left\|u_{j, *}(x)\right\|_{\sigma j, r_{2}}}{\Gamma(1+(s+1) j)} \leqslant & g_{j}+ \\
& \sum_{i \in \mathcal{K}} \sum_{q \in Q_{i}} \sum_{m=0}^{j-v_{i, q}-i} \gamma_{i, q, m} \frac{\left\|u_{j-v_{i, q}-i-m, *}(x)\right\|_{\sigma\left(j-v_{i, q}-i-m\right), r_{2}}}{\Gamma\left(1+(s+1)\left(j-v_{i, q}-i-m\right)\right)}
\end{aligned}
$$

hold for all $j \geqslant 0$ with

$$
g_{j}:=\frac{\left\|f_{j, *}(x)\right\|_{\sigma j, r_{2}}}{\Gamma(1+(s+1) j)}
$$

and

$$
\gamma_{i, q, m}:= \begin{cases}r_{2}^{p-q} \frac{\left\|a_{m, *}^{(i, q)}(x)\right\|_{\sigma\left(v_{i, q}+i+m\right), r_{2}}}{m !} & \text { if } q-p \leqslant 0, \\ (e(v+\kappa))^{q-p} \frac{\left\|a_{m, *}^{(i, q)}(x)\right\|_{\sigma\left(v_{i, q}+i+m\right)-(q-p), r_{2}}}{m !} & \text { if } q-p>0 .\end{cases}
$$

We now shall bound the Nagumo norms $\left\|u_{j, *}(x)\right\|_{\sigma j, r_{2}}$. To do that, we shall proceed as in $[4,42,43]$ by using a technique of majorant series.

Remark 4.19. Like in relation (2.1), some terms $\frac{\left\|u_{j, *}(x)\right\|_{\sigma j, r_{2}}}{\Gamma(1+(s+1) j)}$ may occur in the right-hand side of inequalities (4.9). More precisely such terms exist only for the $q \in$ $Q_{0}$ such that $v_{0, q}=0$ and are obtained when $\left(i, v_{i, q}, m\right)=(0,0,0)$. Consequently, we suppose in the sequel that the positive number $\left.r_{2} \in\right] 0, \rho_{2}[$ has been chosen so that

$$
\sum_{\substack{q \in Q_{0} \\ v_{0, q}=0}} \gamma_{0, q, 0}=\sum_{\substack{q \in Q_{0} \\ v_{0, q}=0}} r_{2}^{p-q}\left\|a_{0, *}^{(0, q)}(x)\right\|_{0, r_{2}}<1 .
$$

Observe that such a choice is already possible since $p-q>0$ for all $q \in Q_{0}$ (see Condition $\left(C_{3}\right)$ ). 
$\triangleleft$ Fourth step: majorant series.

Let us consider the nonnegative numerical sequence $\left(w_{j}\right)$ defined for all $j \geqslant 0$ by the recurrence relations

$$
w_{j}=g_{j}+\sum_{i \in \mathcal{K}} \sum_{q \in Q_{i}} \sum_{m=0}^{j-v_{i, q}-i} \gamma_{i, q, m} w_{j-v_{i, q}-i-m}
$$

where, as previously, the third sum is 0 when $j<v_{i, q}+i$. Observe that the fact that $w_{j} \geqslant 0$ for all $j$ stems from the choice of $r_{2}$ (see Remark 4.19). Observe also we have

$$
0 \leqslant \frac{\left\|u_{j, *}(x)\right\|_{\sigma j, r_{2}}}{\Gamma(1+(s+1) j)} \leqslant w_{j}
$$

for all $j \geqslant 0$ by construction (proceed by induction on $j$ ). Let us now bound the $w_{j}$ 's. To this end, we proceed as follows.

By assumption on the $f_{j, *}$ 's (see the beginning of section 4.3.2), we have

$$
0 \leqslant g_{j} \leqslant \frac{C K^{j} \Gamma(1+(s+1) j)}{\Gamma(1+(s+1) j)} r_{2}^{\sigma j}=C\left(K r_{2}^{\sigma}\right)^{j}
$$

for all $j \geqslant 0$ and the series $g(X):=\sum_{j \geqslant 0} g_{j} X^{j}$ is thereby convergent.

On the other hand, all the terms $a^{(i, q)}(t, x)$ belong to $\mathcal{O}\left(D_{\rho_{2}}\right)\{t\}$. Then, there exist two positive constants $C^{\prime}, K^{\prime}>0$ such that $\left|a_{m, *}^{(i, q)}(x)\right| \leqslant C^{\prime} K^{\prime m} m$ ! for all $i \in\{0, \ldots, \kappa\}, q \in Q_{i}, m \geqslant 0$ and $x \in D_{r_{2}}$. Hence,

$$
0 \leqslant \gamma_{i, q, m} \leqslant \begin{cases}C_{1}^{\prime}\left(K^{\prime} r_{2}^{\sigma}\right)^{m} & \text { if } q-p \leqslant 0, \\ C_{2}^{\prime}\left(K^{\prime} r_{2}^{\sigma}\right)^{m} & \text { if } q-p>0,\end{cases}
$$

with $C_{1}^{\prime}=C^{\prime} r_{2}^{\sigma\left(v_{i, q}+i\right)-(q-p)}=\frac{C_{2}^{\prime}}{(e(v+\kappa))^{q-p}}$ and, thereby, the series $A_{i, q}(X):=$ $\sum_{m \geqslant 0} \gamma_{i, q, m} X^{m}$ are convergent for all $i \in\{0, \ldots, \kappa\}$ and $q \in Q_{i}$.

Consequently, since the series $w(X):=\sum_{j \geqslant 0} w_{j} X^{j}$ satisfies the identity

$$
\left(1-\sum_{i \in \mathcal{K}} \sum_{q \in Q_{i}} X^{v_{i, q}+i} A_{i, q}(X)\right) w(X)=g(X),
$$

it is convergent too. Indeed, since the constant term

$$
1-\sum_{\substack{q \in Q_{0} \\ v_{0, q}=0}} A_{0, q}(0)=1-\sum_{\substack{q \in Q_{0} \\ v_{0, q}=0}} \gamma_{0, q, 0}
$$

is not null by construction (see Remark 4.19), the series $1-\sum_{i \in \mathcal{K}} \sum_{q \in Q_{i}} X^{v_{i, q}+i} A_{i, q}(X)$ is invertible in $\mathbb{C}\{X\}$. Therefore, there exist two positive constants $C^{\prime \prime}, K^{\prime \prime}>0$ such that $w_{j} \leqslant C^{\prime \prime} K^{\prime \prime} j$ for all $j \geqslant 0$. Hence, the following inequalities

$$
\left\|u_{j, *}(x)\right\|_{\sigma j, r_{2}} \leqslant C^{\prime \prime} K^{\prime \prime j} \Gamma(1+(s+1) j)
$$

hold for all $j \geqslant 0$. 
$\triangleleft$ Fifth step: conclusion.

We are left to prove similar estimates on the sup-norm of the $u_{j, *}(x)$ 's. To this end, we proceed by shrinking the domain $D_{r_{2}}$. Let $0<r_{2}^{\prime}<r_{2}$ be. Then, for all $j \geqslant 0$ and $|x| \leqslant r_{2}^{\prime}$, we have

$$
\left|u_{j, *}(x)\right|=\left|u_{j, *}(x) d_{r_{2}}(x)^{\sigma j} \frac{1}{d_{r_{2}}(x)^{\sigma j}}\right| \leqslant \frac{\left|u_{j, *}(x) d_{r_{2}}(x)^{\sigma j}\right|}{\left(r_{2}-r_{2}^{\prime}\right)^{\sigma j}} \leqslant \frac{\left\|u_{j, *}(x)\right\|_{\sigma j, r_{2}}}{\left(r_{2}-r_{2}^{\prime}\right)^{\sigma j}}
$$

and, consequently,

$$
\sup _{|x| \leqslant r_{2}^{\prime}}\left|u_{j, *}(x)\right| \leqslant C^{\prime \prime}\left(\frac{K^{\prime \prime}}{\left(r_{2}-r_{2}^{\prime}\right)^{\sigma}}\right)^{j} \Gamma(1+(s+1) j) .
$$

This achieves the proof of Theorem 4.4.

\section{Summability}

In previous Section 4, we have shown that the formal series solution $\widetilde{u}(t, x)$ and the inhomogeneity $\tilde{f}(t, x)$ of equation (1.2) are together $s$-Gevrey for a convenient $s \geqslant 0$ (see Theorem 4.4). In particular, when $\mathcal{S}=\varnothing$, that is when the Newton polygon $N_{t}(D)$ of the operator $D$ has no side of positive slope, this has allowed us to display a necessary and sufficient condition under which $\widetilde{u}(t, x)$ is convergent (see Corollary 4.5).

In the present section, we are interested in the opposite case $\mathcal{S} \neq \varnothing$, that is in the case where $N_{t}(D)$ has at least one side of positive slope. As previously, we denote by $k$ its smallest positive slope and we set $s=1 / k$. For all $i \in \mathcal{K}$, we also denote by $p_{i}$ the maximum of the $q \in Q_{i}$. Moreover, we assume from now on that equation (1.2) satisfies the four following additional conditions:

$\left(A_{1}\right): p=0$; hence, $\mathcal{K}$ is a non-empty subset of $\{1, \ldots, \kappa\}$,

$\left(A_{2}\right): v_{i, p_{i}}=0$ for all $i \in \mathcal{K}$,

$\left(A_{3}\right): p_{i *}>p_{i}$ for all $i \neq i^{*}$,

$\left(A_{4}\right): a^{\left(i^{*}, p_{i} *\right)}(0,0) \neq 0$.

Observe that Assumptions $\left(A_{1}\right)-\left(A_{2}\right)$ imply $q^{*}=p_{i} *$ and, consequently,

$$
k=\frac{i^{*}}{p_{i *}-i^{*}} \text { and } s=\frac{p_{i *}}{i^{*}}-1
$$

Indeed, the domains $C\left(q-i, v_{i, q}+i\right)$ are included in $C\left(p_{i}-i, i\right)$ for all $i \in \mathcal{K}$ and $q \in Q_{i}$ (see Definition 3.1 for the definition of $N_{t}(D)$ and page 4 for the definition of the domain $C(a, b))$.

Observe also that Assumption $\left(A_{3}\right)$ tells us that $k$ is the unique positive slope of the Newton polygon $N_{t}(D)$.

The aim of this section is to answer to the following question:

$$
\begin{gathered}
\text { "Under Assumptions }\left(A_{1}\right)-\left(A_{4}\right) \text {, how to characterize } \\
\text { the k-summability of } \widetilde{u}(t, x) \text { ?" }
\end{gathered}
$$

A response to this question has already been done by the author in [43] when $i^{*}=\kappa$ the maximum of the $i \in \mathcal{K}$. In the present paper, we consider a much more general situation, where the smallest slope $k>0$ of $N_{t}(D)$ is given by some $i^{*} \leqslant \kappa$ and, in particular, $i^{*}<\kappa$. As we shall see in the sequel, our approach is similar to the one developed in [43], but the calculations are much more complicated because of $i^{*}$ is not necessarily the maximum of the $i \in \mathcal{K}$. 
Before stating our main result (see Theorem 5.4), let us first begin with some recalls about the $k$-summability of formal series in $\mathcal{O}\left(D_{\rho_{2}}\right)[[t]]$.

5.1. $\boldsymbol{k}$-summability. Still considering $t$ as the variable and $x$ as a parameter, one extends, in the similar way as the $s$-Gevrey formal series (see Definition 4.4), the classical notion of $k$-summability of formal series in $\mathbb{C}[[t]]$ to the notion of $k$-summability of formal series in $\mathcal{O}\left(D_{\rho_{2}}\right)[[t]]$ in requiring similar conditions, the estimates being however uniform with respect to $x$. Among the many equivalent definitions of the $k$-summability in a given direction $\arg (t)=\theta$ at $t=0$, we choose here a generalization of Ramis' definition which states that a formal series $\widetilde{g}(t) \in$ $\mathbb{C}[[t]]$ is $k$-summable in the direction $\theta$ if there exists a holomorphic function $g$ which is $s$-Gevrey asymptotic to $\widetilde{g}$ in an open sector $\Sigma_{\theta,>\pi s}$ bisected by $\theta$ and with opening larger than $\pi s$ [40, Def. 3.1]. To express the $s$-Gevrey asymptotic, there also exist various equivalent ways. We choose here the one which sets conditions on the successive derivatives of $g$ (see [25, p. 171] or [40, Thm. 2.4] for instance $)^{4}$.

Definition 5.1 ( $k$-summability). A formal series $\widetilde{u}(t, x) \in \mathcal{O}\left(D_{\rho_{2}}\right)[[t]]$ is said to be $k$-summable in the direction $\arg (t)=\theta$ if there exist a sector $\Sigma_{\theta,>\pi s}$, a radius $0<r_{2}<\rho_{2}$ and a function $u(t, x)$ called $k$-sum of $\widetilde{u}(t, x)$ in the direction $\theta$ such that

(1) $u$ is defined and holomorphic on $\Sigma_{\theta,>\pi s} \times D_{r_{2}+\varepsilon}$ for some $\varepsilon>0$;

(2) for any $|x| \leqslant r_{2}$, the map $t \mapsto u(t, x)$ has $\widetilde{u}(t, x)=\sum_{j \geqslant 0} u_{j, *}(x) \frac{t^{j}}{j !}$ as Taylor series at 0 on $\Sigma_{\theta,>\pi s}$;

(3) for any proper ${ }^{5}$ subsector $\Sigma \Subset \Sigma_{\theta,>\pi s}$, there exist two positive constants $C>0$ and $K>0$ such that, for all $\ell \geqslant 0$ and all $t \in \Sigma$,

$$
\sup _{|x| \leqslant r_{2}}\left|\partial_{t}^{\ell} u(t, x)\right| \leqslant C K^{\ell} \Gamma(1+(s+1) \ell) .
$$

We denote by $\mathcal{O}\left(D_{\rho_{2}}\right)\{t\}_{k ; \theta}$ the subset of $\mathcal{O}\left(D_{\rho_{2}}\right)[[t]]$ made of all the $k$-summable formal series in the direction $\arg (t)=\theta$. Obviously, $\mathcal{O}\left(D_{\rho_{2}}\right)\{t\}_{k ; \theta}$ is included in $\mathcal{O}\left(D_{\rho_{2}}\right)[[t]]_{s}$.

Observe that, for any fixed $x$, the $k$-summability of $\widetilde{u}(t, x)$ coincides with the classical $k$-summability. Consequently, Watson's lemma implies the unicity of its $k$-sum, if any exists.

Observe also that the $k$-sum of a $k$-summable formal series $\widetilde{u}(t, x) \in \mathcal{O}\left(D_{\rho_{2}}\right)\{t\}_{k ; \theta}$ may be analytic with respect to $x$ on a disc smaller than the common disc $D_{\rho_{2}}$ of analyticity of the coefficients $u_{j, *}(x)$ of $\widetilde{u}(t, x)$.

Proposition 5.2 ([43, Prop. 2]). $\left(\mathcal{O}\left(D_{\rho_{2}}\right)\{t\}_{k ; \theta}, \partial_{t}, \partial_{x}\right)$ is a $\mathbb{C}$-differential algebra stable under anti-derivations $\partial_{t}^{-1}$ and $\partial_{x}^{-1}$.

With respect to $t$, the $k$-sum $u(t, x)$ of a $k$-summable series $\widetilde{u}(t, x) \in \mathcal{O}\left(D_{\rho_{2}}\right)\{t\}_{k ; \theta}$ is analytic on an open sector for which there is no control on the angular opening except that it must be larger than $\pi s$ (hence, it contains a closed sector $\bar{\Sigma}_{\theta, \pi s}$ bisected by $\theta$ and with opening $\pi s$ ) and no control on the radius except that it

\footnotetext{
${ }^{4}$ In Appendix A page 31, we present various results of the general theory of the Gevrey asymptotic expansions in the framework of the formal power series in $\mathcal{O}\left(D_{\rho_{2}}\right)[[t]]$.

${ }^{5}$ A subsector $\Sigma$ of a sector $\Sigma^{\prime}$ is said to be a proper subsector and one denotes $\Sigma \Subset \Sigma^{\prime}$ if its closure in $\mathbb{C}$ is contained in $\Sigma^{\prime} \cup\{0\}$.
} 
must be positive. Thereby, the $k$-sum $u(t, x)$ is well-defined as a section of the sheaf of analytic functions in $(t, x)$ on a germ of closed sector of opening $\pi s$ (that is, a closed interval $\bar{I}_{\theta, \pi s}$ of length $\pi s$ on the circle $S^{1}$ of directions issuing from 0 ; see $[26,1.1]$ or $[15$, I.2]) times $\{0\}$ (in the plane $\mathbb{C}$ of the variable $x$ ). We denote by $\mathcal{O}_{\bar{I}_{\theta, \pi s} \times\{0\}}$ the space of such sections.

Corollary 5.3. The operator of k-summation

$$
\begin{aligned}
& \mathcal{S}_{k ; \theta}: \mathcal{O}\left(D_{\rho_{2}}\right)\{t\}_{k ; \theta} \longrightarrow \mathcal{O}_{\bar{I}_{\theta, \pi s} \times\{0\}} \\
& \widetilde{u}(t, x) \quad \longmapsto u(t, x)
\end{aligned}
$$

is a homomorphism of $\mathbb{C}$-differential algebras for the derivations $\partial_{t}$ and $\partial_{x}$. Moreover, it commutes with the anti-derivations $\partial_{t}^{-1}$ and $\partial_{x}^{-1}$.

Let us now turn to the study of our formal series solution $\widetilde{u}(t, x)$.

\subsection{Main result.}

5.2.1. A preliminary remark. Before stating the main result of this section, let us first begin with a preliminary remark on the series $\widetilde{u}(t, x)$. According to Notation 1.1 , let us write the coefficients $a^{(i, q)}(t, x)$ on the form $a^{(i, q)}(t, x)=\sum_{n \geqslant 0} a_{*, n}^{(i, q)}(t) \frac{x^{n}}{n !}$ with $a_{*, n}^{(i, q)}(t) \in \mathcal{O}\left(D_{\rho_{1}}\right)$ for all $i \in \mathcal{K}, q \in Q_{i}$ and $n \geqslant 0$. Then, an identification of the powers in $x$ in the equation

$$
D\left(\sum_{n \geqslant 0} \widetilde{u}_{*, n}(t) \frac{x^{n}}{n !}\right)=\sum_{n \geqslant 0} \tilde{f}_{*, n}(t) \frac{x^{n}}{n !}
$$

provides for all $n \geqslant 0$ the recurrence relations

$$
\begin{aligned}
a_{*, 0}^{\left(i^{*}, p_{i *}\right)}(t) \partial_{t}^{-i^{*}} \widetilde{u}_{*, n+p_{i}}(t) & =\widetilde{u}_{*, n}(t)-\widetilde{f}_{*, n}(t) \\
-\sum_{m=1}^{n}\left(\begin{array}{c}
n \\
m
\end{array}\right) & a_{*, m}^{\left(i^{*}, p_{i} *\right)}(t) \partial_{t}^{-i^{*}} \widetilde{u}_{*, n-m+p_{i} *}(t) \\
& \quad-\sum_{i \in \mathcal{K}} \sum_{q \in \mathcal{Q}_{i}} \sum_{m=0}^{n}\left(\begin{array}{c}
n \\
m
\end{array}\right) t^{v_{i, q}} a_{*, m}^{(i, q)}(t) \partial_{t}^{-i} \widetilde{u}_{*, n-m+q}(t)
\end{aligned}
$$

where the $\mathcal{Q}_{i}$ 's are defined by $\mathcal{Q}_{i *}=Q_{i *} \backslash\left\{p_{i}\right\}$ and $\mathcal{Q}_{i}=Q_{i}$ if $i \neq i^{*}$. In particular, these relations tell us that each $\widetilde{u}_{*, \ell}(t)$ (hence, $\widetilde{u}(t, x)$ too) is uniquely determined from $\tilde{f}(t, x)$ and from the $\widetilde{u}_{*, n}(t)$ with $n=0, \ldots, p_{i^{*}}-1$. Indeed, Assumption $\left(A_{3}\right)$ implies $q<p_{i}$ * for all $i \in \mathcal{K}$ and $q \in \mathcal{Q}_{i}$, and Assumption $\left(A_{4}\right)$ implies that the quotient $1 / a_{*, 0}^{\left(i^{*}, p_{i}\right)}(t)$ is well-defined in $\mathbb{C}[[t]]$.

5.2.2. Main result. We are now able to state the main result in view in this section.

Theorem 5.4. Let a direction $\arg (t)=\theta$ issuing from 0 be given. Then,

(1) The formal series $\widetilde{u}(t, x) \in \mathcal{O}\left(D_{\rho_{2}}\right)[[t]]$ is $k$-summable in the direction $\theta$ if and only if the inhomogeneity $\widetilde{f}(t, x)$ and the $p_{i}$ coefficients $\widetilde{u}_{*, n}(t) \in \mathbb{C}[[t]]$ with $n \in\left\{0, \ldots, p_{i *}-1\right\}$ are $k$-summable in the direction $\theta$.

(2) Moreover, the $k$-sum $u(t, x)$ in the direction $\theta$, if any exists, satisfies equation (1.2) in which $\tilde{f}(t, x)$ is replaced by its $k$-sum $f(t, x)$ in the direction $\theta$. 
Remark 5.5. The necessary condition of Point 1 is straigthforward from Proposition 5.2. Indeed, we have $\widetilde{u}_{*, n}(t)=\partial_{x}^{n} \widetilde{u}(t, x)_{\mid x=0}$ and $\widetilde{f}=D \widetilde{u}$. Moreover, Point 2 stems obvious from Corollary 5.3. Thereby, we are left to prove the sufficient condition of Point 1.

Remark 5.6. Theorem 5.4 generalizes the results of summability already proved by W. Balser and M. Loday-Richaud in [4] and by the author in [42,43].

5.3. Proof of Theorem 5.4. As we said in Remark 5.5 just above, it remains to prove the sufficient condition of Point 1. Consequently, we fix from now on a direction $\theta$ and we suppose that the inhomogeneity $\widetilde{f}(t, x)$ and the coefficients $\tilde{u}_{*, n}(t)$ for $n \in\left\{0, \ldots, p_{i_{-1}}\right\}$ are all $k$-summable in this direction.

$\triangleleft$ First step: the associated equation.

Let us first begin by introducing the functions $b^{(i, q)}(t, x)$ defined, for all $i \in \mathcal{K}$ and $q \in Q_{i}$, by

$$
b^{(i, q)}(t, x)= \begin{cases}\frac{1}{a^{\left(i^{*}, p_{i} *\right)}(t, x)} & \text { if }(i, q)=\left(i^{*}, p_{i}\right), \\ \frac{t^{v_{i, q}} a^{(i, q)}(t, x)}{a^{\left(i^{*}, p_{i}\right)}(t, x)} & \text { if }(i, q) \neq\left(i^{*}, p_{i}\right) .\end{cases}
$$

Thanks to Assumption $\left(A_{4}\right)$, all these functions are holomorphic on a common domain $D_{\rho_{1}^{\prime}} \times D_{\rho_{2}^{\prime}}$ of $(0,0) \in \mathbb{C}^{2}$ for two suitable radiuses $\rho_{1}^{\prime}, \rho_{2}^{\prime}>0$.

Let us now write $\widetilde{u}(t, x)$ on the form

$$
\widetilde{u}(t, x)=\sum_{n=0}^{p_{i *}-1} \widetilde{u}_{*, n}(t) \frac{x^{n}}{n !}+\partial_{x}^{-p_{i} *} \widetilde{v}(t, x)
$$

with $\widetilde{v}(t, x) \in \mathcal{O}\left(D_{\rho_{2}}\right)[[t]]$ and let us set $\widetilde{w}:=\partial_{t}^{-i^{*}} \widetilde{v}$. Then, equation (1.2) becomes

$$
\Delta \widetilde{w}=\widetilde{g}(t, x),
$$

where $\Delta$ is the linear integro-differential operator

$$
\Delta:=1-b^{\left(i^{*}, p_{i *}\right)}(t, x) \partial_{x}^{-p_{i} *} \partial_{t}^{i^{*}}+\sum_{i \in \mathcal{K}} \sum_{q \in \mathcal{Q}_{i}} b^{(i, q)}(t, x) \partial_{x}^{q-p_{i} *} \partial_{t}^{i^{*}-i}
$$

and where the inhomogeneity $\widetilde{g}(t, x)$ is defined by

$$
\begin{aligned}
\widetilde{g}(t, x):=b^{\left(i^{*}, p_{i *}\right)}(t, x)\left(\sum_{n=0}^{p_{i *}-1} \tilde{u}_{*, n}(t)\right. & \left.\frac{x^{n}}{n !}-\widetilde{f}(t, x)\right) \\
& -\sum_{i \in \mathcal{K}} \sum_{q \in \mathcal{Q}_{i}} \sum_{n=0}^{p_{i *-1-q}} b^{(i, q)}(t, x) \widetilde{u}_{*, n+q}(t) \frac{x^{n}}{n !} .
\end{aligned}
$$

Indeed, we have $\partial_{t}^{i^{*}-i} \partial_{t}^{-i^{*}}=\partial_{t}^{-i}$ for all $i \in \mathcal{K}$. The sets $\mathcal{Q}_{i}$ are the sets introduced in the preliminary remark of Section 5.2: $\mathcal{Q}_{i^{*}}=Q_{i *} \backslash\left\{p_{i *}\right\}$ and $\mathcal{Q}_{i}=Q_{i}$ if $i \neq i^{*}$.

According to our assumption (see the beginning of Section 5.3) and Proposition 5.2 , the inhomogeneity $\widetilde{g}(t, x)$ of equation (5.2) is $k$-summable in the direction $\theta$. Thereby, to prove our result, it suffices to prove that the formal power series $\widetilde{w}(t, x) \in \mathcal{O}\left(D_{\rho_{2}}\right)[[t]]$ is also $k$-summable in the direction $\theta$. To do that, we shall proceed similarly as in $[4,42,43]$ by using a standard fixed point procedure. 
$\triangleleft$ Second step: the fixed point procedure.

Let us set $\widetilde{w}(t, x)=\sum_{m \geqslant 0} \widetilde{w}_{m}(t, x)$ and let us consider the solution of equation (5.2), where the $\widetilde{w}_{m}(t, x)$ 's belong to $\mathcal{O}\left(D_{\rho}\right)[[t]]$ for a suitable common $\rho>0$ and are recursively determined, for all $m \geqslant 0$, by the relations

$$
\left\{\begin{array}{l}
\widetilde{w}_{0}=\tilde{g} \\
\widetilde{w}_{m+1}=b^{\left(i^{*}, p_{i} *\right)}(t, x) \partial_{x}^{-p_{i *}} \partial_{t}^{i^{*}} \widetilde{w}_{m}-\sum_{i \in \mathcal{K}} \sum_{q \in \mathcal{Q}_{i}} b^{(i, q)}(t, x) \partial_{x}^{q-p_{i} *} \partial_{t}^{i^{*}-i} \widetilde{w}_{m}
\end{array}\right.
$$

Observe that, for all $m \geqslant 0$, the formal series $\widetilde{w}_{m}(t, x)$ are of order $O\left(x^{m}\right)$ in $x$ and, consequently, the series $\widetilde{w}(t, x)$ itself makes sense as a formal series in $t$ and $x$. Indeed, the definition of the $\mathcal{Q}_{i}$ 's and Assumption $\left(A_{3}\right)$ imply $q-p_{i} * 0$ for all $i \in \mathcal{K}$ and $q \in \mathcal{Q}_{i}$.

Let us now denote by $w_{0}(t, x)$ the $k$-sum of $\widetilde{w}_{0}=\widetilde{g}$ in the direction $\theta$ and, for all $m \geqslant 0$, let $w_{m}(t, x)$ be determined as the solution of system (5.3) in which all the $\widetilde{w}_{m}$ are replaced by $w_{m}$. By construction, all the $w_{m}(t, x)$ are defined and holomorphic on a common domain $\Sigma_{\theta,>\pi s} \times D_{\rho_{2}^{\prime \prime}}$, where the radius $\rho_{1}^{\prime \prime}$ of $\Sigma_{\theta,>\pi s}$ and the radius $\rho_{2}^{\prime \prime}$ of $D_{\rho_{2}^{\prime \prime}}$ can always be chosen so that $0<\rho_{1}^{\prime \prime}<\min \left(1, \rho_{1}^{\prime}\right)$ and $0<\rho_{2}^{\prime \prime}<\min \left(1, \rho_{2}, \rho_{2}^{\prime}\right)$.

To end the proof, it remains to prove that the series $\sum_{m \geqslant 0} w_{m}(t, x)$ is convergent and that its sum $w(t, x)$ is the $k$-sum of $\widetilde{w}(t, x)$ in the direction $\theta$.

$\triangleleft$ Third step: some estimations on $w_{m}(t, x)$.

According to Definition 5.1, the $k$-summability of $\widetilde{w}_{0}=\widetilde{g}$ implies that there exists a radius $0<r_{2}<\rho_{2}^{\prime \prime}$ with the following property: for any proper subsector $\Sigma \Subset \Sigma_{\theta,>\pi s}$, there exist two positive constants $C>0$ and $K>0$ such that, for all $\ell \geqslant 0$ and all $(t, x) \in \Sigma \times D_{r_{2}}$, the function $w_{0}$ satisfies the conditions

$$
\left|\partial_{t}^{\ell} w_{0}(t, x)\right| \leqslant C K^{\ell} \Gamma(1+(s+1) \ell) .
$$

Let us now fix a proper subsector $\Sigma \Subset \Sigma_{\theta,>\pi s}$. Let $r_{1}$ denote the radius of $\Sigma$ and let us choose for the constant $K$ of the previous property a constant $\geqslant$ $\max \left(1, \frac{1}{\rho_{1}^{\prime \prime}-r_{1}}\right)$. Observe that such a choice is already possible since conditions (5.4) still hold for any constant $K^{\prime} \geqslant K$. Observe also that the quotient $1 /\left(\rho_{1}^{\prime \prime}-r_{1}\right)$ makes sense since the definition of a proper subsector (see Footnote 5) implies $0<r_{1}<\rho_{1}^{\prime \prime}$.

Proposition 5.7. Let us denote by

- $I:=\left\lfloor\frac{\kappa}{i^{*}}\right\rfloor$, where $\lfloor\cdot\rfloor$ denotes the lower integer part of $\cdot$,

- $B:=\max _{\substack{i \in \mathcal{K} \\ q \in Q_{i}}}\left(\max _{(t, x) \in \bar{D}_{\rho_{1}^{\prime \prime}} \times \bar{D}_{\rho_{2}^{\prime \prime}}}\left|b^{(i, q)}(t, x)\right|\right)$ the maximum of the functions $\left|b^{(i, q)}(t, x)\right|$ on $\bar{D}_{\rho_{1}^{\prime \prime}} \times \bar{D}_{\rho_{2}^{\prime \prime}}$, where $\bar{D}_{\rho}$ denotes the closed disc with center 0 and radius $\rho>0$,

- $B^{\prime}:=(\kappa+1)\left(I i^{*}+1\right) B$. 
Let $\left(P_{m}(x)\right)$ be the sequence of polynomials in $\mathbb{R}^{+}[x]$ recursively determined by

$$
\left\{\begin{array}{l}
P_{0}(x)=1, \\
P_{m+1}(x)=\left(\partial_{x}^{-p_{i} *}+\sum_{i \in \mathcal{K}^{\prime}} \sum_{q \in \mathcal{Q}_{i}^{\prime}} \frac{\left(m p_{i *}\right) !}{\left(m p_{i *}+p_{i}\right) !} \partial_{x}^{-q}\right) P_{m}(x) \quad \text { for } m \geqslant 0,
\end{array}\right.
$$

with $\mathcal{K}^{\prime}:=\left\{i \in \mathcal{K} ; p_{i} \geqslant 1\right\}$ and $\mathcal{Q}_{i}^{\prime}:=\left\{\max \left(p_{i^{*}}-p_{i}, 1\right), \ldots, p_{i *}-1\right\}$. Then, the following inequalities

$$
\left|\partial_{t}^{\ell} w_{m}(t, x)\right| \leqslant C B^{\prime m} K^{i^{*} m+\ell} \Gamma\left(1+(s+1)\left(i^{*} m+\ell\right)\right) P_{m}(|x|)
$$

hold for all $m, \ell \geqslant 0$ and all $(t, x) \in \Sigma \times D_{r_{2}}$.

Remark 5.8. Since $1 \leqslant i^{*} \leqslant \kappa$, we have $1 \leqslant I \leqslant \kappa$ and $I i^{*} \geqslant 1$. More precisely, and thanks to the definition of the lower interger part, we have $I i^{*}>\kappa-i^{*}$.

Remark 5.9. The constant $B$ is well-defined since the functions $b^{(i, q)}(t, x)$ are all holomorphic on $D_{\rho_{1}^{\prime}} \times D_{\rho_{2}^{\prime}}$ and the radiuses $\rho_{j}^{\prime \prime}$ satisfy $0<\rho_{j}^{\prime \prime}<\rho_{j}^{\prime}$ for $j=1,2$.

Remark 5.10. The set $\mathcal{K}^{\prime}$ already contains $i^{*}$ and, therefore, is never empty. We have indeed the inequalities $p_{i *}>i^{*} \geqslant 1$.

Proof. The proof proceeds by recursion on $m \geqslant 0$.

The case $m=0$ is straightforward from inequality (5.4). Let us now suppose that inequalities (5.5) hold for a certain $m \geqslant 0$.

From identities (5.3) and the Leibniz formula, we first derive the inequalities

$$
\begin{aligned}
\left|\partial_{t}^{\ell} w_{m+1}(t, x)\right| \leqslant \sum_{j=0}^{\ell} & \left(\begin{array}{l}
\ell \\
j
\end{array}\right)\left|\partial_{t}^{\ell-j} b^{\left(i^{*}, p_{i} *\right)}(t, x)\right|\left|\partial_{x}^{-p_{i} *} \partial_{t}^{i^{*}+j} w_{m}(t, x)\right| \\
& +\sum_{i \in \mathcal{K}} \sum_{q \in \mathcal{Q}_{i}} \sum_{j=0}^{\ell}\left(\begin{array}{l}
\ell \\
j
\end{array}\right)\left|\partial_{t}^{\ell-j} b^{(i, q)}(t, x)\right|\left|\partial_{x}^{q-p_{i} *} \partial_{t}^{i^{*}-i+j} w_{m}(t, x)\right|
\end{aligned}
$$

for all $\ell \geqslant 0$ and $(t, x) \in \Sigma \times D_{r_{2}}$. On the other hand, for all $i \in \mathcal{K}, q \in Q_{i}, k \geqslant 0$ and $(t, x) \in \Sigma \times D_{r_{2}}$, the Cauchy integral formula allows us to write the derivative $\partial_{t}^{k} b^{(i, q)}(t, x)$ on the form

$$
\partial_{t}^{k} b^{(i, q)}(t, x)=\frac{k !}{(2 i \pi)^{2}} \int_{\substack{\left|t^{\prime}-t\right|=\rho_{1}^{\prime \prime}-r_{1} \\\left|x^{\prime}-x\right|=\rho_{2}^{\prime \prime}-r_{2}}} \frac{b^{(i, q)}\left(t^{\prime}, x^{\prime}\right)}{\left(t^{\prime}-t\right)^{k+1}\left(x^{\prime}-x\right)} d t^{\prime} d x^{\prime}
$$

(we have indeed $0<r_{1}<\rho_{1}^{\prime \prime}$ and $0<r_{2}<\rho_{2}^{\prime \prime}$ ), which yields the estimates

$$
\left|\partial_{t}^{k} b^{(i, q)}(t, x)\right| \leqslant k ! B\left(\frac{1}{\rho_{1}^{\prime \prime}-r_{1}}\right)^{k} \leqslant k ! B K^{k}
$$

Hence, according to the fact that $0<r_{1}<1$ and $K \geqslant 1$, the following inequalities: for all $\ell \geqslant 0$ and $(t, x) \in \Sigma \times D_{r_{2}}$,

$$
\left|\partial_{t}^{\ell} w_{m+1}(t, x)\right| \leqslant C B B^{\prime m} K^{i^{*}(m+1)+\ell} \sum_{i \in \mathcal{K} \cup\{0\}}\left(S_{i, \ell, m} \sum_{q \in \mathcal{Q}_{i}}\left(\partial_{x}^{q-p_{i} *} P_{m}\right)(|x|)\right),
$$

where we set $\mathcal{Q}_{0}:=\left\{p_{0}=0\right\}$ and where $S_{i, \ell, m}$ is the sum defined by: 
- Case $i \in\left\{0, \ldots, i^{*}\right\}$ :

$$
S_{i, \ell, m}:=\sum_{j=0}^{\ell} \frac{\ell !}{j !} \Gamma\left(1+(s+1)\left(i^{*} m+i^{*}-i+j\right)\right) .
$$

- Case $i \in\left\{i^{*}+1, \ldots, \kappa\right\}$ :

$S_{i, \ell, m}:=\sum_{j=0}^{J_{i, i *, \ell}} \frac{\ell !}{j !} \Gamma\left(1+(s+1) i^{*} m\right)+\sum_{j=J_{i, i} *, \ell}^{\ell} \frac{\ell !}{j !} \Gamma\left(1+(s+1)\left(i^{*} m+i^{*}-i+j\right)\right)$,

with $J_{i, i *, \ell}=\min \left(i-i^{*}-1, \ell\right)$. Of course, the second sum is zero as soon as $J_{i, i *, \ell}=\ell$, that is $\ell \leqslant i-i^{*}-1$.

Applying then Lemma 5.11 below, we get

$$
\begin{aligned}
\left|\partial_{t}^{\ell} w_{m+1}(t, x)\right| \leqslant C\left(I i^{*}+1\right) B B^{\prime m} & K^{i^{*}(m+1)+\ell} \Gamma\left(1+(s+1)\left(i^{*}(m+1)+\ell\right)\right) \\
& \times \sum_{i \in \mathcal{K} \cup\{0\}} \sum_{q \in \mathcal{Q}_{i}} \frac{\left(m p_{i *}\right) !}{\left(m p_{i *}+p_{i}\right) !}\left(\partial_{x}^{q-p_{i} *} P_{m}\right)(|x|)
\end{aligned}
$$

and inequalities (5.5) follow by observing that the double-sum of the right-hand side satisfies

$$
\begin{aligned}
\sum_{i \in \mathcal{K} \cup\{0\}} \sum_{q \in \mathcal{Q}_{i}} \frac{\left(m p_{i *}\right) !}{\left(m p_{i}+p_{i}\right) !}\left(\partial_{x}^{q-p_{i} *} P_{m}\right)(|x|) & \leqslant(\kappa+1)\left(\partial_{x}^{-p_{i} *} P_{m}\right)(|x|) \\
& +\sum_{i \in \mathcal{K}^{\prime}} \sum_{q \in \mathcal{Q}_{i}^{\prime}} \frac{\left(m p_{i *}\right) !}{\left(m p_{i *}+p_{i}\right) !}\left(\partial_{x}^{-q} P_{m}\right)(|x|) ;
\end{aligned}
$$

hence,

$$
\sum_{i \in \mathcal{K} \cup\{0\}} \sum_{q \in \mathcal{Q}_{i}} \frac{\left(m p_{i} *\right) !}{\left(m p_{i *}+p_{i}\right) !}\left(\partial_{x}^{q-p_{i} *} P_{m}\right)(|x|) \leqslant(\kappa+1) P_{m+1}(|x|) .
$$

Indeed, $\mathcal{K} \subset\{1, \ldots, \kappa\}$, the coefficients of the polynomial $P_{m}$ are positive and the quotients $\left(m p_{i *}\right) ! /\left(m p_{i} *+p_{i}\right)$ ! are $\leqslant 1$ for all $i \in \mathcal{K}$. This ends the proof of Proposition 5.7.

Lemma 5.11. Let $i \in \mathcal{K} \cup\{0\}, \ell \geqslant 0$ and $m \geqslant 0$ be. Then,

$$
S_{i, \ell, m} \leqslant\left(I i^{*}+1\right) \frac{\left(m p_{i *}\right) !}{\left(m p_{i *}+p_{i}\right) !} \Gamma\left(1+(s+1)\left(i^{*}(m+1)+\ell\right)\right) .
$$

This technical lemma will be proved later in Section 5.4. For the moment, let us end the proof of Theorem 5.4.

The following proposition, already proved in [43], allows to bound the $P_{m}(|x|)$ 's.

Proposition 5.12 ([43, Prop. 5]). Let $m \geqslant 0$ be. Then,

$$
P_{m}(|x|) \leqslant \frac{\left(\kappa p_{i *} 2^{p_{i *}}\left(1+p_{i *}\right)^{p_{i *}-1}\right)^{m}}{\left(m p_{i *}\right) !}|x|^{m}
$$

for all $x \in D_{r_{2}}$. 
Let us set $B^{\prime \prime}:=B^{\prime} K^{i^{*}} \kappa p_{i *} 2^{p_{i} *}\left(1+p_{i *}\right)^{p_{i *-1}}$. Then, Propositions 5.7 and 5.12 imply, for all $\ell \geqslant 0$ and $(t, x) \in \Sigma \times D_{r_{2}}$, the inequalities

$$
\left|\partial_{t}^{\ell} w_{m}(t, x)\right| \leqslant C K^{\ell} \Gamma\left(1+(s+1)\left(i^{*} m+\ell\right)\right) \frac{\left(B^{\prime \prime}|x|\right)^{m}}{\left(m p_{i}\right) !}
$$

hence, the inequalities

$$
\left|\partial_{t}^{\ell} w_{m}(t, x)\right| \leqslant C\left(2^{p_{i} * K}\right)^{\ell} \Gamma(1+(s+1) \ell)\left(2^{p_{i} * B^{\prime \prime}}|x|\right)^{m} .
$$

Indeed, the conditions $(s+1) i^{*}=p_{i *}$ and $s+1 \leqslant p_{i *}$ (see relations (5.1)) imply

$$
\begin{aligned}
\Gamma\left(1+(s+1)\left(i^{*} m+\ell\right)\right) & =\Gamma\left(1+(s+1) \ell+m p_{i^{*}}\right) \\
& =\Gamma(1+(s+1) \ell) \prod_{j=1}^{m p_{i}^{*}}((s+1) \ell+j) \\
& \leqslant \Gamma(1+(s+1) \ell) \prod_{j=1}^{m p_{i}^{*}}\left(\ell p_{i^{*}}+j\right) \\
& =\Gamma(1+(s+1) \ell) \frac{\left(\ell p_{i *}+m p_{i^{*}}\right) !}{\left(\ell p_{i^{*}}\right) !}
\end{aligned}
$$

and, consequently,

$$
\begin{aligned}
\frac{\Gamma\left(1+(s+1)\left(i^{*} m+\ell\right)\right)}{\left(m p_{i *}\right) !} & \leqslant \Gamma(1+(s+1) \ell)\left(\begin{array}{c}
\ell p_{i *}+m p_{i *} \\
m p_{i *}
\end{array}\right) \\
& \leqslant 2^{\ell p_{i *} * m p_{i} *} \Gamma(1+(s+1) \ell) .
\end{aligned}
$$

We are now able to complete the proof of Theorem 5.4.

$\triangleleft$ Fourth step: conclusion.

Let us choose for $\Sigma$ a sector containing a proper subsector $\Sigma^{\prime}$ bisected by the direction $\theta$ and opening larger than $\pi s$ (such a choice is already possible by definition of a proper subsector, see Footnote 5 ).

Let us also choose $r, \varepsilon>0$ so that $0<r<r+\varepsilon<\min \left(r_{2}, 2^{-p_{i} *} / B^{\prime \prime}\right)$ and let us set $C^{\prime}:=C \sum_{m \geqslant 0}\left(2^{p_{i} *} B^{\prime \prime} r\right)^{m} \in \mathbb{R}^{+}$and $K^{\prime}:=2^{p_{i} *} K$.

Thanks to inequalities (5.7), the series $\sum_{m \geqslant 0} \partial_{t}^{\ell} w_{m}(t, x)$ are normally convergent on $\Sigma \times D_{r+\varepsilon}$ for all $\ell \geqslant 0$ and satisfy the inequalities

$$
\sum_{m \geqslant 0}\left|\partial_{t}^{\ell} w_{m}(t, x)\right| \leqslant C^{\prime} K^{\prime \ell} \Gamma(1+(s+1) \ell)
$$

for all $(t, x) \in \Sigma \times D_{r+\varepsilon}$. In particular, the sum $w(t, x)$ of the series $\sum_{m \geqslant 0} w_{m}(t, x)$ is well-defined, holomorphic on $\Sigma \times D_{r+\varepsilon}$ and satisfies the inequalities

$$
\left|\partial_{t}^{\ell} w(t, x)\right| \leqslant C^{\prime} K^{\ell} \Gamma(1+(s+1) \ell)
$$

for all $\ell \geqslant 0$ and $(t, x) \in \Sigma \times D_{r+\varepsilon}$. Hence, Conditions 1 and 3 of Definition 5.1.

To prove the second condition of Definition 5.1, we proceed as follows. The removable singularities theorem implies the existence of $\lim _{\substack{t \rightarrow 0 \\ t \in \Sigma^{\prime}}} \partial_{t}^{\ell} w(t, x)$ for all $x \in \bar{D}_{r}$ and, thereby, the existence of the Taylor series of $w$ at 0 on $\Sigma^{\prime}$ for all $x \in \bar{D}_{r}$ (see for instance [25, Cor. 1.1.3.3]; see also [16, Prop. 1.1.11]). On the other hand, 
considering recurrence relations (5.3) with $w_{m}$ and the $k$-sum $g(t, x)$ instead of $\widetilde{w}_{m}$ and $\widetilde{g}(t, x)$, it is clear that $w(t, x)$ satisfies equation (5.2) with right-hand side $g(t, x)$ in place of $\widetilde{g}(t, x)$ and, consequently, so does its Taylor series. Then, since equation (5.2) has a unique formal series solution $\widetilde{w}(t, x)$ (proceed similarly as Theorem 2.1 by exchanging the roles of $x$ and $t$ ), we then conclude that the Taylor expansion of $w(t, x)$ is $\widetilde{w}(t, x)$. Hence, Condition 2 of Definition 5.1 .

This achieves the proof of the $k$-summability of $\widetilde{w}(t, x)$ and, thereby, the sufficient condition of point 1 of Theorem 5.4.

5.4. Proof of Lemma 5.11. We are left to prove the technical Lemma 5.11. Before starting the calculations, let us first recall a classical result on the Gamma function which will be us useful in the sequel.

Lemma 5.13. Let $a$ and $b$ be two nonnegative numbers satisfying $b \geqslant \max (2,1+a)$. Then, $\Gamma(1+a) \leqslant \Gamma(b)$.

Proof. Lemma 5.13 stems from the increase of the Gamma function on $[2,+\infty$ [ and from the fact that $\Gamma(c) \leqslant 1=\Gamma(2)$ for all $c \in[1,2]$. Indeed, we have the inequalities $2 \leqslant 1+a \leqslant b$ for all $a \geqslant 1$ and the inequalities $1 \leqslant 1+a \leqslant 2 \leqslant b$ for all $a \in[0,1]$.

Let us also recall that, according to our assumptions $\left(A_{1}\right)$ and $\left(A_{2}\right)$, Lemma 4.10 implies the following inequalities

$$
(s+1) i \geqslant p_{i} \quad \text { for all } i \in \mathcal{K} .
$$

Observe that these latter still hold when $i=0$ since $p_{0}=0$.

As we shall see below, inequalities (5.8) will play a crucial role in our proof.

Let us now prove Lemma 5.11.

$\triangleleft$ First case. When $i \in\left\{0, \ldots, i^{*}\right\}$, inequalities (5.6) are a consequence of the three following lemmas.

Lemma 5.14. Let $\ell \geqslant 0, j \in\{0, \ldots, \ell\}$ and $m \geqslant 0$ be. Then,

$$
\frac{\ell !}{j !} \Gamma\left(1+(s+1)\left(i^{*} m+i^{*}-i+j\right)\right) \leqslant \Gamma\left(1+(s+1)\left(i^{*} m+i^{*}-i+j\right)+\ell-j\right) .
$$

Proof. Lemma 5.14 is clear when $j=\ell$ and stems obvious from the inequality

$$
\frac{\ell !}{j !}=\prod_{n=1}^{\ell-j}(j+n) \leqslant \prod_{n=1}^{\ell-j}\left((s+1)\left(i^{*} m+i^{*}-i+j\right)+n\right)
$$

and from the relation

$$
\begin{aligned}
& \Gamma\left(1+(s+1)\left(i^{*} m+i^{*}-i+j\right)+\ell-j\right)= \\
& \Gamma\left(1+(s+1)\left(i^{*} m+i^{*}-i+j\right)\right) \prod_{n=1}^{\ell-j}\left((s+1)\left(i^{*} m+i^{*}-i+j\right)+n\right)
\end{aligned}
$$

when $j<\ell$.

Lemma 5.15. Let $\ell \geqslant 0$ and $m \geqslant 0$ be. Then,

$$
\sum_{j=0}^{\ell} \frac{\Gamma\left(1+(s+1)\left(i^{*} m+i^{*}-i+j\right)+\ell-j\right)}{\Gamma\left(1+(s+1)\left(i^{*} m+i^{*}-i+\ell\right)\right)} \leqslant I i^{*}+1 .
$$


Proof. $\star$ Let us first suppose $\ell \leqslant I i^{*}$. Inequality (5.9) is clear when $(\ell, m, i)=$ $\left(0,0, i^{*}\right)$. Otherwise, we have

$$
\begin{aligned}
1+\underbrace{(s+1)\left(i^{*} m+i^{*}-i+j\right)+\ell-j}_{\geqslant 0} & =1+(s+1)\left(i^{*} m+i^{*}-i\right)+\ell+s j \\
& \leqslant \underbrace{1+(s+1)\left(i^{*} m+i^{*}-i+\ell\right)}_{\geqslant 2}
\end{aligned}
$$

for all $j \in\{0, \ldots, \ell\}$, and inequality (5.9) stems from Lemma 5.13:

$$
\sum_{j=0}^{\ell} \frac{\Gamma\left(1+(s+1)\left(i^{*} m+i^{*}-i+j\right)+\ell-j\right)}{\Gamma\left(1+(s+1)\left(i^{*} m+i^{*}-i+\ell\right)\right)} \leqslant \sum_{j=0}^{\ell} 1=\ell+1 \leqslant I i^{*}+1 .
$$

* Let us now suppose $\ell>I i^{*}$ and let us write the sum of (5.9) on the form

$$
\sum_{j=0}^{\ell}(\ldots)=\sum_{j=0}^{\ell-I i^{*}}(\ldots)+\sum_{j=\ell-I i^{*}+1}^{\ell}(\ldots) .
$$

By similarly to the previous case, we get

$$
\sum_{j=\ell-I i^{*}+1}^{\ell}(\ldots) \leqslant \sum_{j=\ell-I i^{*}+1}^{\ell} 1=I i^{*}
$$

On the other hand, we have the inequalities

$$
\begin{aligned}
2 & \leqslant 1+I i^{*} \\
& \leqslant 1+(s+1)\left(i^{*} m+i^{*}-i+j\right)+\ell-j \\
& \leqslant 1+(s+1)\left(i^{*} m+i^{*}-i+\ell\right)-s I i^{*} \\
& \leqslant 1+(s+1)\left(i^{*} m+i^{*}-i+\ell\right)-1
\end{aligned}
$$

for all $j \in\left\{0, \ldots, \ell-I i^{*}\right\}$. Indeed, the relation $(s+1) i^{*}=p_{i *}$ (see (5.1)) and the definition of $i^{*}$ (see Remark 3.4) imply

$$
s I i^{*}=I\left((s+1) i^{*}-i^{*}\right)=I\left(p_{i}-i^{*}\right) \geqslant I \geqslant 1 .
$$

Consequently, by applying Lemma 5.13, the first sum of the right-hand side of (5.10) is bounded as follows:

$$
\begin{aligned}
\sum_{j=0}^{\ell-I i^{*}}(\ldots) & \leqslant\left(\ell-I i^{*}+1\right) \frac{\Gamma\left(1+(s+1)\left(i^{*} m+i^{*}-i+\ell\right)-s I i^{*}\right)}{\Gamma\left(1+(s+1)\left(i^{*} m+i^{*}-i+\ell\right)\right)} \\
& =\frac{\ell-I i^{*}+1}{(s+1)\left(i^{*} m+i^{*}-i+\ell\right)} \times \frac{\Gamma\left(1+(s+1)\left(i^{*} m+i^{*}-i+\ell\right)-s I i^{*}\right)}{\Gamma\left(1+(s+1)\left(i^{*} m+i^{*}-i+\ell\right)-1\right)} \\
& \leqslant \frac{\ell-I i^{*}+1}{(s+1)\left(i^{*} m+i^{*}-i+\ell\right)} .
\end{aligned}
$$

Inequality (5.9) follows then by observing that

$$
\frac{\ell-I i^{*}+1}{(s+1)\left(i^{*} m+i^{*}-i+\ell\right)} \leqslant \frac{1}{s+1} \leqslant 1
$$

for all $\ell>I i^{*}$. This ends the proof of Lemma 5.15. 
Lemma 5.16. Let $\ell \geqslant 0$ and $m \geqslant 0$ be. Then,

$$
\Gamma\left(1+(s+1)\left(i^{*} m+i^{*}-i+\ell\right)\right) \leqslant \frac{\left(m p_{i}\right) !}{\left(m p_{i}+p_{i}\right) !} \Gamma\left(1+(s+1)\left(i^{*}(m+1)+\ell\right)\right) .
$$

Proof. Thanks to the relations (5.8), we have the inequalities

$$
\begin{aligned}
\underbrace{1+(s+1)\left(i^{*}(m+1)+\ell\right)}_{\geqslant 2} & =1+(s+1)\left(i^{*} m+i^{*}-i+\ell\right)+(s+1) i \\
& \geqslant 1+\underbrace{(s+1)\left(i^{*} m+i^{*}-i+\ell\right)+p_{i}}_{\geqslant 0} .
\end{aligned}
$$

Hence, the inequalities

$$
\Gamma\left(1+(s+1)\left(i^{*}(m+1)+\ell\right)\right) \geqslant \Gamma\left(1+(s+1)\left(i^{*} m+i^{*}-i+\ell\right)+p_{i}\right)
$$

by applying Lemma 5.13 . Lemma 5.16 is then proved when $p_{i}=0$ and follows from the identity

$$
\begin{aligned}
& \Gamma\left(1+(s+1)\left(i^{*} m+i^{*}-i+\ell\right)+p_{i}\right)= \\
& \Gamma\left(1+(s+1)\left(i^{*} m+i^{*}-i+\ell\right)\right) \prod_{n=1}^{p_{i}}\left((s+1)\left(i^{*} m+i^{*}-i+\ell\right)+n\right)
\end{aligned}
$$

and from the relations

$$
\begin{aligned}
\prod_{n=1}^{p_{i}}\left((s+1)\left(i^{*} m+i^{*}-i+\ell\right)+n\right) & \geqslant \prod_{n=1}^{p_{i}}\left((s+1) i^{*} m+n\right) \quad\left(\text { since } i^{*}-i \geqslant 0\right) \\
& =\prod_{n=1}^{p_{i}}\left(m p_{i}+n\right) \quad\left(\text { since }(s+1) i^{*}=p_{i}\right) \\
& =\frac{\left(m p_{i}+p_{i}\right) !}{\left(m p_{i}\right) !}
\end{aligned}
$$

when $p_{i} \geqslant 1$.

$\triangleleft$ Second case. When $i \in\left\{i^{*}+1, \ldots, \kappa\right\}^{6}$, Lemma 5.11 is proved in a similar way as the previous case. However, the calculations are much more complicated because of the term $J_{i, i^{*}, \ell}=\min \left(i-i^{*}-1, \ell\right)$ and of the fact that $i^{*}-i$ is negative.

Lemma 5.17. Let $\ell \geqslant 0, j \in\{0, \ldots, \ell\}$ and $m \geqslant 0$ be. Then,

$$
\frac{\ell !}{j !} \Gamma\left(1+(s+1) i^{*} m\right) \leqslant \Gamma\left(1+(s+1)\left(i^{*} m+j\right)+\ell-j\right) .
$$

Moreover, if $i-i^{*} \leqslant j \leqslant \ell$, then

$$
\begin{aligned}
& \frac{\ell !}{j !} \Gamma\left(1+(s+1)\left(i^{*} m+i^{*}-i+j\right)\right) \leqslant \\
& \quad \Gamma\left(1+(s+1)\left(i^{*} m+i^{*}-i+j\right)+\ell-j+i-i^{*}\right) .
\end{aligned}
$$

Proof. Lemma 5.17 is proved in a similar way as Lemma 5.14 by respectively using the relation

$$
\Gamma\left(1+(s+1) i^{*} m\right) \leqslant \Gamma\left(1+(s+1)\left(i^{*} m+j\right)\right)
$$

\footnotetext{
${ }^{6}$ Of course, this case occurs if and only if $i^{*}<\kappa$.
} 
for inequality (5.11) and the relations

$$
\begin{aligned}
\frac{\ell !}{j !}=\prod_{n=1}^{\ell-j}(j+n) & =\prod_{n=1}^{\ell-j}(\underbrace{i^{*}-i+j}_{\geqslant 0}+n+\underbrace{i-i^{*}}_{>0}) \\
& =\prod_{n=1+i-i^{*}}^{\ell-j+i-i^{*}}\left(i^{*}-i+j+n\right) \\
& \leqslant \prod_{n=1}^{\ell-j+i-i^{*}}\left(i^{*}-i+j+n\right) \\
& \leqslant \prod_{n=1}^{\ell-j+i-i^{*}}\left((s+1)\left(i^{*} m+i^{*}-i+j\right)+n\right)
\end{aligned}
$$

for inequality (5.12). Observe that the two conditions $j \geqslant i-i^{*}$ and $i-i^{*}>0$ play a key role in these various calculations.

Lemma 5.18. Let $\ell \geqslant 0$ and $m \geqslant 0$ be. Then,

$$
\sum_{j=0}^{J_{i, i}^{*}, \ell} \frac{\Gamma\left(1+(s+1)\left(i^{*} m+j\right)+\ell-j\right)}{\Gamma\left(1+(s+1)\left(i^{*} m+\ell\right)\right)} \leqslant i-i^{*} .
$$

Moreover, if $\ell \geqslant i-i^{*}$ (hence, $\left.J_{i, i} *, \ell=i-i^{*}-1\right)$, then

$$
\sum_{j=i-i^{*}}^{\ell} \frac{\Gamma\left(1+(s+1)\left(i^{*} m+i^{*}-i+j\right)+\ell-j+i-i^{*}\right)}{\Gamma\left(1+(s+1)\left(i^{*} m+\ell\right)\right)} \leqslant I i^{*}+i^{*}-i+1 .
$$

Proof. * Inequality (5.13) is clear when $m=\ell=0$ (we have indeed $J_{i, i, \ell}=0$; hence, $j=0$ too) and stems from the relations

$$
1+\underbrace{(s+1)\left(i^{*} m+j\right)+\ell-j}_{\geqslant 0}=1+(s+1) i^{*} m+\ell+s j \leqslant \underbrace{1+(s+1)\left(i^{*} m+\ell\right)}_{\geqslant 2}
$$

and Lemma 5.13 otherwise. Indeed, we have in this case

$$
\sum_{j=0}^{J_{i, i} * \ell} \frac{\Gamma\left(1+(s+1)\left(i^{*} m+j\right)+\ell-j\right)}{\Gamma\left(1+(s+1)\left(i^{*} m+\ell\right)\right)} \leqslant \sum_{j=0}^{J_{i, i} *, \ell} 1=J_{i, i *, \ell}+1 \leqslant i-i^{*} .
$$

* Let us now prove inequality (5.14) and let us suppose for the moment that $\ell \in$ $\left\{i-i^{*}, \ldots, I i^{*}\right\}^{7}$. Then, the condition $\ell \geqslant i-i^{*}>0$ implies

$$
\begin{aligned}
1+\underbrace{(s+1)\left(i^{*} m+i^{*}-i+j\right)+\ell-j+i-i^{*}}_{\geqslant 0} & =1+(s+1) i^{*} m+\ell+s(\underbrace{i^{*}-i+j}_{\leqslant j \leqslant \ell}) \\
& \leqslant \underbrace{1+(s+1)\left(i^{*} m+\ell\right)}_{\geqslant 2}
\end{aligned}
$$

\footnotetext{
${ }^{7}$ This set makes sense since, thanks to Remark 9, we have $I i^{*}>\kappa-i^{*} \geqslant i-i^{*}$.
} 
for all $j \in\left\{i-i^{*}, \ldots, \ell\right\}$ and inequality (5.14) follows from Lemma 5.13:

$$
\begin{aligned}
\sum_{j=i-i^{*}}^{\ell} \frac{\Gamma\left(1+(s+1)\left(i^{*} m+i^{*}-i+j\right)+\ell-j+i-i^{*}\right)}{\Gamma\left(1+(s+1)\left(i^{*} m+\ell\right)\right)} & \leqslant \sum_{j=i-i^{*}}^{\ell} 1 \\
& =\ell+i^{*}-i+1 \\
& \leqslant I i^{*}+i^{*}-i+1 .
\end{aligned}
$$

When $\ell>I i^{*}$, we proceed similarly as in Lemma 5.15 by writing the sum of (5.14) on the form

$$
\sum_{j=i-i^{*}}^{\ell}(\ldots)=\sum_{j=i-i^{*}}^{\ell-I i^{*}+i-i^{*}}(\ldots)+\sum_{j=\ell-I i^{*}+i-i^{*}+1}^{\ell}(\ldots)
$$

and by observing that the two sums of the right-hand side can be respectively bounded as follows:

$$
\sum_{j=i-i^{*}}^{\ell-I i^{*}+i-i^{*}}(\ldots) \leqslant 1 \quad \text { and } \quad \sum_{j=\ell-I i *+i-i^{*}+1}^{\ell}(\ldots) \leqslant I i^{*}+i^{*}-i .
$$

The first inequality is proved as in Lemma 5.15 by using Lemma 5.13 and the relations

$$
\begin{aligned}
2 & \leqslant 1+I i^{*} \\
& \leqslant 1+(s+1)\left(i^{*} m+i^{*}-i+j\right)+\ell-j+i-i^{*} \\
& =1+(s+1) i^{*} m+\ell+s\left(i^{*}-i+j\right) \\
& \leqslant 1+(s+1)\left(i^{*} m+\ell\right)-s I i^{*} \\
& \leqslant 1+(s+1)\left(i^{*} m+\ell\right)-1
\end{aligned}
$$

for all $j \in\left\{i-i^{*}, \ldots, \ell-I i^{*}+i-i^{*}\right\}$. As for the second inequality, it stems from Lemma 5.13 and the relation

$$
1+(s+1)\left(i^{*} m+i^{*}-i+j\right)+\ell-j+i-i^{*} \leqslant 1+(s+1)\left(i^{*} m+\ell\right)
$$

proved just above. This ends the proof of Lemma 5.18.

Let us now apply Lemmas 5.17 and 5.18:

- Case $\ell \leqslant i-i^{*}-1$. Then,

$$
S_{i, \ell, m} \leqslant\left(i-i^{*}\right) \Gamma\left(1+(s+1)\left(i^{*} m+\ell\right)\right) \leqslant\left(I i^{*}+1\right) \Gamma\left(1+(s+1)\left(i^{*} m+\ell\right)\right) .
$$

Indeed, the second sum of $S_{i, \ell, m}$ is zero (we have $J_{i, i *, \ell}=\ell$ ) and Remark 9 implies $I i^{*}>\kappa-i^{*} \geqslant i-i^{*}$.

- Case $\ell \geqslant i-i^{*}$. Then,

$$
\begin{aligned}
S_{i, \ell, m} & \leqslant\left(i-i^{*}+I i^{*}-i+i^{*}+1\right) \Gamma\left(1+(s+1)\left(i^{*} m+\ell\right)\right) \\
& =\left(I i^{*}+1\right) \Gamma\left(1+(s+1)\left(i^{*} m+\ell\right)\right) .
\end{aligned}
$$

Thereby, to end the proof of Lemma 5.11, we are left to prove the following.

Lemma 5.19. Let $\ell \geqslant 0$ and $m \geqslant 0$ be. Then,

$$
\Gamma\left(1+(s+1)\left(i^{*} m+\ell\right)\right) \leqslant \frac{\left(m p_{i *}\right) !}{\left(m p_{i}+p_{i}\right) !} \Gamma\left(1+(s+1)\left(i^{*}(m+1)+\ell\right)\right) .
$$


Proof. Using the relation $(s+1) i^{*}=p_{i *}$ (see (5.1)) and the fact that $p_{i *}$ is the maximum of the $p_{i}$ (see Assumption $\left(A_{3}\right)$ ), we successively have

$$
\begin{aligned}
\underbrace{1+(s+1)\left(i^{*}(m+1)+\ell\right)}_{\geqslant 2} & =1+(s+1)\left(i^{*} m+\ell\right)+p_{i} * \\
& \geqslant 1+\underbrace{(s+1)\left(i^{*} m+\ell\right)+p_{i}}_{\geqslant 0}
\end{aligned}
$$

and the inequalities

$$
\Gamma\left(1+(s+1)\left(i^{*}(m+1)+\ell\right)\right) \geqslant \Gamma\left(1+(s+1)\left(i^{*} m+\ell\right)+p_{i}\right)
$$

by applying Lemma 5.13. Then, we conclude as in Lemma 5.16.

\section{Appendix A. Gevrey asymptotic}

In this appendix, we present various results of the general theory of the Gevrey asymptotic expansions in the framework of the formal power series in $\mathcal{O}\left(D_{\rho_{2}}\right)[[t]]$.

A.1. $s$-Gevrey asymptotic. Still considering $t$ as the variable and $x$ as a parameter, one extends, in the similar way as the $s$-Gevrey formal series (see Definition 4.1), the classical notion of Gevrey asymptotic to a formal series in $\mathbb{C}[[t]]$ to the one of Gevrey asymptotic to a formal series in $\mathcal{O}\left(D_{\rho_{2}}\right)[[t]]$ in requiring similar conditions, the estimates being however uniform with respect to $x$.

Definition A.1 (s-Gevrey asymptotic). Let $s \geqslant 0$ and $\Sigma$ be an open sector with vertex $0 \in \mathbb{C}$. A function $u(t, x)$ holomorphic on a domain $\Sigma \times D_{\rho}$ for some $\rho>0$ is said to be Gevrey asymptotic of order $s$ (in short, $s$-Gevrey asymptotic) to a formal series $\sum_{j \geqslant 0} u_{j, *}(x) \frac{t^{j}}{j !} \in \mathcal{O}\left(D_{\rho_{2}}\right)[[t]]$ on $\Sigma$ if there exists $0<r_{2}<\min \left(\rho, \rho_{2}\right)$ such that, for any proper subsector $\Sigma^{\prime} \Subset \Sigma$, there exist two positive constants $C>0$ and $K>0$ such that, for all $J \geqslant 1$ and all $t \in \Sigma^{\prime}$ :

$$
\sup _{|x| \leqslant r_{2}}\left|u(t, x)-\sum_{j=0}^{J-1} u_{j, *}(x) \frac{t^{j}}{j !}\right| \leqslant C K^{J} \Gamma(1+s J)|t|^{J} .
$$

A series which is the $s$-Gevrey asymptotic expansion of a function is said to be an $s$-Gevrey asymptotic series on $\Sigma$.

Remark A.2. If any exists, the $s$-Gevrey asymptotic series is unique.

Proposition A.3. Let $s \geqslant 0$ be. Then, a $s$-Gevrey asymptotic series on a sector $\Sigma$ is a $s$-Gevrey series.

Proof. Let $\widetilde{u}(t, x)=\sum_{j \geqslant 0} u_{j, *}(x) \frac{t^{j}}{j !} \in \mathcal{O}\left(D_{\rho_{2}}\right)[[t]]$ be a $s$-Gevrey asymptotic series of a function $u(t, x)$ on $\Sigma$. We want to prove that there exist positive constants $0<r_{2}^{\prime \prime}<\rho_{2}, C^{\prime \prime}>0$ and $K^{\prime \prime}>0$ such that, for all $J \geqslant 0$,

$$
\sup _{|x| \leqslant r_{2}^{\prime \prime}}\left|u_{J, *}(x)\right| \leqslant C^{\prime \prime} K^{\prime \prime J} \Gamma(1+(s+1) J) .
$$


Let $r_{2}>0$ be as in Definition A.1 and let us choose $\Sigma^{\prime} \Subset \Sigma$ a proper subsector of $\Sigma$. For any $J \geqslant 1$, we derive from condition (A.1) applied twice to the relation

$$
u_{J, *}(x) \frac{t^{J}}{J !}=\left(u(t, x)-\sum_{j=0}^{J-1} u_{j, *}(x) \frac{t^{j}}{j !}\right)-\left(u(t, x)-\sum_{j=0}^{J} u_{j, *}(x) \frac{t^{j}}{j !}\right)
$$

the following inequality

$$
\sup _{|x| \leqslant r_{2}}\left|u_{J, *}(x)\right| \leqslant C K^{J} \Gamma(1+s J) J !+C K^{J+1} \Gamma(1+s(J+1)) J ! R,
$$

where $R>0$ denotes the radius of $\Sigma^{\prime}$. Applying then the relation between the Gamma and the Beta functions to $\Gamma(1+s J) J !=\Gamma(1+s J) \Gamma(1+J)$, we get

$$
\Gamma(1+s J) J !=\Gamma(2+(s+1) J) \int_{0}^{1} t^{s J}(1-t)^{J} d t \leqslant \Gamma(2+(s+1) J)
$$

hence, the inequalities

$$
\Gamma(1+s J) J ! \leqslant(1+(s+1) J) \Gamma(1+(s+1) J) \leqslant e\left(e^{s+1}\right)^{J} \Gamma(1+(s+1) J) .
$$

In the same way, and using besides the increase of the Gamma function on $[2,+\infty[$, we have

$$
\Gamma(1+s(J+1)) J ! \leqslant \Gamma(2+(s+1) J+s) \leqslant \Gamma(2+(s+1) J+S),
$$

where $S$ is an integer $\geqslant s$; hence,

$$
\Gamma(1+s(J+1)) J ! \leqslant \Gamma(1+(s+1) J) \prod_{\ell=1}^{S+1}((s+1) J+\ell) \leqslant A B^{J} \Gamma(1+(s+1) J)
$$

with convenient constants $A, B>0$ independent of $J$. Consequently, there exist $C^{\prime}, K^{\prime}>0$ such that the following inequalities

$$
\sup _{|x| \leqslant r_{2}}\left|u_{J, *}(x)\right| \leqslant C^{\prime} K^{\prime J} \Gamma(1+(s+1) J)
$$

hold for all $J \geqslant 1$. Condition (A.2) follows then by choosing

$$
r_{2}^{\prime \prime}=r_{2}, C^{\prime \prime}=\max \left(C^{\prime}, \sup _{|x| \leqslant r_{2}}\left|u_{0, *}(x)\right|\right) \text { and } K^{\prime \prime}=K^{\prime}
$$

This ends the proof.

Following Proposition A.4 gives us a characterization of the $s$-Gevrey asymptotic in terms of conditions on the successive derivatives $\partial_{t}^{J} u$ of the function $u$ with respect to $t$.

Proposition A.4. Let $s \geqslant 0$ and $\Sigma$ be an open sector with vertex $0 \in \mathbb{C}$. Then, a function $u(t, x)$ holomorphic on a domain $\Sigma \times D_{\rho}$ for some $\rho>0$ is s-Gevrey asymptotic to a formal series $\widetilde{u}(t, x)=\sum_{j \geqslant 0} u_{j, *}(x) \frac{t^{j}}{j !} \in \mathcal{O}\left(D_{\rho_{2}}\right)[[t]]$ on $\Sigma$ if and only if there exists $0<r_{2}<\min \left(\rho, \rho_{2}\right)$ such that

(1) for any $|x| \leqslant r_{2}$, the map $t \mapsto u(t, x)$ has $\widetilde{u}(t, x)$ as Taylor series at 0 on $\Sigma$, 
(2) for any proper subsector $\Sigma^{\prime} \Subset \Sigma$, there exist two positive cConstants $C>0$ and $K>0$ such that, for all $J \geqslant 0$ and all $t \in \Sigma^{\prime}$,

$$
\sup _{|x| \leqslant r_{2}}\left|\partial_{t}^{J} u(t, x)\right| \leqslant C K^{J} \Gamma(1+(s+1) J) .
$$

Proof. $\triangleleft$ Necessary condition. Let us suppose that $u(t, x)$ is $s$-Gevrey asymptotic to $\widetilde{u}(t, x)$ on $\Sigma$ and let us prove Conditions 1 and 2 of Proposition A.4.

Due to Definition A.1, Condition 1 is straightforward. To prove Ccondition 2, we consider $0<r_{2}<\min \left(\rho, \rho_{2}\right)$ as in Definition A.1 and a proper subsector $\Sigma^{\prime} \Subset \Sigma$ and we choose a radius $0<r_{2}^{\prime}<r_{2}$, a sector $\Sigma^{\prime \prime}$ such that $\Sigma^{\prime} \Subset \Sigma^{\prime \prime} \Subset \Sigma$ and a positive constant $\delta>0$ small enough so that, for all $t \in \Sigma^{\prime}$, the closed disc centered at $t$ with radius $|t| \delta$ be contained in $\Sigma^{\prime \prime}$. Then, the Cauchy integral formula implies

$$
\begin{aligned}
\partial_{t}^{J} u(t, x) & =\frac{J !}{(2 i \pi)^{2}} \int_{\substack{\left|t^{\prime}-t\right|=|t| \delta \\
\left|x^{\prime}-x\right|=r_{2}-r_{2}^{\prime}}} \frac{u\left(t^{\prime}, x^{\prime}\right)}{\left(t^{\prime}-t\right)^{J+1}\left(x^{\prime}-x\right)} d t^{\prime} d x^{\prime} \\
& =\frac{J !}{(2 i \pi)^{2}} \int_{\substack{\left|t^{\prime}-t\right|=|t| \delta \\
\left|x^{\prime}-x\right|=r_{2}-r_{2}^{\prime}}}\left(u\left(t^{\prime}, x^{\prime}\right)-\sum_{j=0}^{J-1} u_{j, *}(x) \frac{t^{j}}{j !}\right) \frac{d t^{\prime} d x^{\prime}}{\left(t^{\prime}-t\right)^{J+1}\left(x^{\prime}-x\right)}
\end{aligned}
$$

for all $J \geqslant 0$, all $t \in \Sigma^{\prime}$ and all $|x| \leqslant r_{2}^{\prime}$. Indeed, the sum is 0 when $J=0$ and the $J$-th derivative of a polynomial of degree $J-1$ is 0 too when $J \geqslant 1$. Hence,

$$
\begin{aligned}
\left|\partial_{t}^{J} u(t, x)\right| & \leqslant \frac{J !}{(2 \pi)^{2}} \sup _{\substack{\left|t^{\prime}-t\right|=|t| \delta \\
\left|x^{\prime}-x\right|=r_{2}-r_{2}^{\prime}}}\left|u\left(t^{\prime}, x^{\prime}\right)\right| \frac{(2 \pi)^{2}}{(|t| \delta)^{J}} \\
& \leqslant C K^{J} \Gamma(1+s J) J ! \frac{|t|^{J}(1+\delta)^{J}}{|t|^{J} \delta^{J}} \\
& \leqslant C^{\prime} K^{\prime J} \Gamma(1+(s+1) J)
\end{aligned}
$$

with $C^{\prime}=e C$ and $K^{\prime}=e^{s+1} K\left(1+\frac{1}{\delta}\right)$. Indeed, we have previously saw in the proof of Proposition A.3 that $\Gamma(1+s J) J ! \leqslant e^{1+(s+1) J} \Gamma(1+(s+1) J)$. This proves Condition 2 and, consequently, the necessary condition.

$\triangleleft$ Sufficient condition. Let us now suppose that Conditions 1 and 2 are satisfied and let us prove condition (A.1) of Definition A.1. To do that, let us consider a proper subsector $\Sigma^{\prime} \Subset \Sigma$.

For any fixed $|x| \leqslant r_{2}$, the map $t \mapsto u(t, x)$ admits the Taylor expansion with integral remainder

$$
u(t, x)-\sum_{j=0}^{J-1} \frac{\partial^{j} u}{\partial t^{j}}\left(t_{0}, x\right) \frac{\left(t-t_{0}\right)^{j}}{j !}=\int_{t_{0}}^{t} \frac{\left(t-t^{\prime}\right)^{J-1}}{(J-1) !} \frac{\partial^{J} u}{\partial t^{J}}\left(t^{\prime}, x\right) d t^{\prime}
$$

for all $J \geqslant 1$, all $t \in \Sigma^{\prime}$ and all $t_{0} \in \Sigma^{\prime}$. Due to Condition $1, \lim _{\substack{t_{0} \rightarrow 0 \\ t_{0} \in \Sigma^{\prime}}} \frac{\partial^{j} u}{\partial t^{j}}\left(t_{0}, x\right)$ exists for all $j \geqslant 0$ and is equal to $u_{j, *}(x)$. Therefore, the limits of the left-hand and of 
the right-hand sides of (A.3) both exist when $t_{0} \rightarrow 0$ and we have

$$
u(t, x)-\sum_{j=0}^{J-1} u_{j, *}(x) \frac{t^{j}}{j !}=\int_{0}^{t} \frac{\left(t-t^{\prime}\right)^{J-1}}{(J-1) !} \frac{\partial^{J} u}{\partial t^{J}}\left(t^{\prime}, x\right) d t^{\prime}
$$

for all $J \geqslant 1$, all $t \in \Sigma^{\prime}$ and all $|x| \leqslant r_{2}$. Hence, applying Condition 2 :

$$
\sup _{|x| \leqslant r_{2}}\left|u(t, x)-\sum_{j=0}^{J-1} u_{j, *}(x) \frac{t^{j}}{j !}\right| \leqslant \sup _{\substack{t^{\prime} \in \Sigma^{\prime} \\|x| \leqslant r_{2}}}\left|\frac{\partial^{J} u}{\partial t^{J}}\left(t^{\prime}, x\right)\right| \frac{|t|^{J}}{J !} \leqslant C K^{J} \frac{\Gamma(1+(s+1) J)}{J !}|t|^{J}
$$

for all $J \geqslant 1$ and all $t \in \Sigma^{\prime}$. Condition (A.3) follows then from the inequality

$$
\frac{\Gamma(1+(s+1) J)}{J !} \leqslant 2^{(S+1) J} \Gamma(1+s J) \quad, S \in \mathbb{N}, S \geqslant s
$$

which stems from the relations

$$
\Gamma(1+(s+1) J)=\Gamma(1+s j) \prod_{j=1}^{J}(s J+j) \leqslant \Gamma(1+s J) \prod_{j=1}^{J}(S J+j)
$$

and

$$
\frac{\prod_{j=1}^{J}(S J+j)}{J !}=\left(\begin{array}{c}
(S+1) J \\
J
\end{array}\right) \leqslant \sum_{k=0}^{(S+1) J}\left(\begin{array}{c}
(S+1) J \\
k
\end{array}\right)=2^{(S+1) J} .
$$

This proves the sufficient condition; hence, Proposition A.4.

In the sequel, we denote by

- $\overline{\mathcal{A}}_{s}\left(\Sigma, D_{\rho_{2}}\right)$ the set of all the functions which are $s$-Gevrey asymptotic on $\Sigma$ to a formal series of $\mathcal{O}\left(D_{\rho_{2}}\right)[[t]]$

- $T_{s ; \Sigma, D_{\rho_{2}}}: \overline{\mathcal{A}}_{s}\left(\Sigma, D_{\rho_{2}}\right) \longrightarrow \mathcal{O}\left(D_{\rho_{2}}\right)[[t]]_{s}$ the map which assigns to each $u(t, x) \in \overline{\mathcal{A}}_{s}\left(\Sigma, D_{\rho_{2}}\right)$ its $s$-Gevrey asymptotic series.

Observe that $T_{s ; \Sigma, D_{\rho_{2}}}$ is well-defined due to Remark A.2 and Proposition A.4. Following Proposition A.5 specifies the algebraic properties of $\overline{\mathcal{A}}_{s}\left(\Sigma, D_{\rho_{2}}\right)$ and $T_{s ; \Sigma, D_{\rho_{2}}}$.

Proposition A.5. Let $s \geqslant 0$ and $\Sigma$ be an open sector with vertex $0 \in \mathbb{C}$.

(1) $\left(\overline{\mathcal{A}}_{s}\left(\Sigma, D_{\rho_{2}}\right), \partial_{t}, \partial_{x}\right)$ is a $\mathbb{C}$-differential algebra stable under the anti-derivations $\partial_{t}^{-1}$ and $\partial_{x}^{-1}$.

(2) The map $T_{s ; \Sigma, D_{\rho_{2}}}: \overline{\mathcal{A}}_{s}\left(\Sigma, D_{\rho_{2}}\right) \longrightarrow \mathcal{O}\left(D_{\rho_{2}}\right)[[t]]_{s}$ is a homomorphism of $\mathbb{C}$ differential algebras for the derivations $\partial_{t}$ and $\partial_{x}$. Moreover, it commutes with the anti-derivations $\partial_{t}^{-1}$ and $\partial_{x}^{-1}$.

Proof. The proof is the same that the one given in [43, Prop. 2].

\section{A.2. The $s$-Gevrey Borel-Ritt Theorem.}

Theorem A.6. Supposons that $\Sigma$ has opening $\leqslant \pi s$. Then, the map $T_{s ; \Sigma, D_{\rho_{2}}}$ is onto.

Proof. It is sufficient to consider a sector $\Sigma$ with opening $\pi s$. Moreover, by means of a rotation, we can besides assume that $\Sigma$ is bisected by the direction $\theta=0$. We denote by $R$ its radius. 
$\star$ Let $\widetilde{u}(t, x)=\sum_{j \geqslant 0} u_{j, *}(x) \frac{t^{j}}{j !} \in \mathcal{O}\left(D_{\rho_{2}}\right)[[t]]_{s}$ a $s$-Gevrey formal series. By assumption, the coefficients $u_{j, *}(x)$ satisfy the following two conditions:

- $u_{j, *}(x) \in \mathcal{O}\left(D_{\rho_{2}}\right)$ for all $j \geqslant 0$,

- there exist $0<r_{2}<\rho_{2}, C>0$ and $K>0$ such that $\left|u_{j, *}(x)\right| \leqslant C K^{j} \Gamma(1+$ $(s+1) j)$ for all $j \geqslant 0$ and $|x| \leqslant r_{2}$.

Therefore, the series $\widehat{u}(\tau, x)=\sum_{j \geqslant 0} \frac{u_{j, *}(x) \tau^{j}}{\Gamma(1+s j) j !}$ converges for all $(\tau, x) \in D_{\rho} \times D_{r_{2}}$, where $\rho$ is the radius of convergence of $\sum_{j \geqslant 0} \frac{\Gamma(1+(s+1) j)}{\Gamma(1+s j) j !}(K \tau)^{j}$.

$\star$ Let us now fix $b \in D_{\rho}, b>0$, and let us consider the holomorphic function $u(t, x) \in \mathcal{O}\left(\Sigma \times D_{r_{2}}\right)$ defined by

$$
u(t, x)=t^{-k} \int_{0}^{b^{k}} \widehat{u}\left(\xi^{s}, x\right) e^{-\xi / t^{k}} d \xi, \text { where } s=\frac{1}{k} \text { and } \xi=\tau^{k} .
$$

We shall prove below that $u(t, x)$ is $s$-Gevrey asymptotic to $\widetilde{u}(t, x)$ on $\Sigma$.

$\star$ Let $0<r_{2}^{\prime}<r_{2}$. For any $0<\delta<\frac{\pi}{2}$ and $0<R^{\prime}<R$, we denote by $\Sigma_{\delta}$ the proper subsector of $\Sigma$ defined by

$$
\Sigma_{\delta}=\left\{t \in \mathbb{C} ;|\arg (t)|<\frac{\pi}{2 k}-\frac{\delta}{k} \text { and } 0<|t|<R^{\prime}\right\} .
$$

Let $J \geqslant 1$ and $(t, x) \in \Sigma_{\delta} \times \bar{D}_{r_{2}^{\prime}}$ be. From the relation

$$
t^{j}=t^{-k} \int_{0}^{+\infty} \frac{\xi^{s j}}{\Gamma(1+s j)} e^{-\xi / t^{k}} d \xi, j \geqslant 0
$$

(see [2, pp. 78-79] for instance), we first have

$$
\begin{aligned}
u(t, x)-\sum_{j=0}^{J-1} u_{j, *}(x) \frac{t^{j}}{j !}=t^{-k} \int_{0}^{b^{k}}( & \left.\sum_{j \geqslant 0} \frac{u_{j, *}(x)}{\Gamma(1+s j) j !} \xi^{s j} e^{-\xi / t^{k}}\right) d \xi \\
& -\sum_{j=0}^{J-1} \frac{u_{j, *}(x)}{j !} t^{-k} \int_{0}^{+\infty} \frac{\xi^{s j}}{\Gamma(1+s j)} e^{-\xi / t^{k}} d \xi .
\end{aligned}
$$

Since

$$
t \in \Sigma_{\delta} \Rightarrow|\arg (t)|<\frac{\pi}{2} \Rightarrow \Re(t)>0 \Rightarrow\left|\xi^{s j} e^{-\xi / t^{k}}\right|=|\xi|^{s j} e^{-\xi \frac{\Re\left(t^{k}\right)}{|t|^{2 k}}} \leqslant b^{j}
$$

for all $\xi \in\left[0, b^{k}\right]$, the series $\sum_{j \geqslant 0} \frac{u_{j, *}(x)}{\Gamma(1+s j) j !} \xi^{s j} e^{-\xi / t^{k}}$ converges normally on $\left[0, b^{k}\right]$.

Therefore, we can permute the sum and the integral. Hence,

$$
\begin{aligned}
u(t, x)-\sum_{j=0}^{J-1} u_{j, *}(x) \frac{t^{j}}{j !}=\sum_{j \geqslant J} \frac{u_{j, *}(x)}{\Gamma(1+s j) j !} t^{-k} \int_{0}^{b^{k}} \xi^{s j} e^{-\xi / t^{k}} d \xi & \\
& -\sum_{j=0}^{J-1} \frac{u_{j, *}(x)}{\Gamma(1+s j) j !} t^{-k} \int_{b^{k}}^{+\infty} \xi^{s j} e^{-\xi / t^{k}} d \xi .
\end{aligned}
$$


Let us now observe that the inequalities $\left(\xi / b^{k}\right)^{s j} \leqslant\left(\xi / b^{k}\right)^{J s}$ hold both when $\xi \leqslant b^{k}$ and $j \geqslant J$ and when $\xi \geqslant b^{k}$ and $j<J$. This brings then us to the following

$$
\begin{aligned}
\left|u(t, x)-\sum_{j=0}^{J-1} u_{j, *}(x) \frac{t^{j}}{j !}\right| & \leqslant \sum_{j \geqslant J} \frac{b^{j-J}\left|u_{j, *}(x)\right|}{\Gamma(1+s j) j !}|t|^{-k} \int_{0}^{b^{k}} \xi^{s J} e^{-\xi \Re\left(1 / t^{k}\right)} d \xi \\
& +\sum_{j=0}^{J-1} \frac{b^{j-J}\left|u_{j, *}(x)\right|}{\Gamma(1+s j) j !}|t|^{-k} \int_{b^{k}}^{+\infty} \xi^{s j} e^{-\xi \Re\left(1 / t^{k}\right)} d \xi \\
& \leqslant \sum_{j \geqslant 0} \frac{b^{j-J}\left|u_{j, *}(x)\right|}{\Gamma(1+s j) j !}|t|^{-k} \int_{0}^{+\infty} \xi^{s j} e^{-\xi \Re\left(1 / t^{k}\right)} d \xi \\
& \leqslant \sum_{j \geqslant 0} \frac{b^{j-J}\left|u_{j, *}(x)\right|}{\Gamma(1+s j) j !}|t|^{-k} \int_{0}^{+\infty} \xi^{s j} e^{\left.-\xi \sin (\delta) /|t|^{k}\right)} d \xi .
\end{aligned}
$$

Observe that the last inequality stems from the fact that $t \in \Sigma_{\delta}$ implies

$$
\Re\left(\frac{1}{t^{k}}\right)=\frac{\cos \left(\arg \left(t^{k}\right)\right)}{|t|^{k}} \geqslant \frac{\cos \left(\frac{\pi}{2}-\delta\right)}{|t|^{k}}=\frac{\sin (\delta)}{|t|^{k}} .
$$

Setting then $u=\frac{\xi \sin (\delta)}{|t|^{k}}$, we obtain

$$
\begin{aligned}
\left|u(t, x)-\sum_{j=0}^{J-1} u_{j, *}(x) \frac{t^{j}}{j !}\right| & \leqslant \sum_{j \geqslant 0} \frac{b^{j-J}\left|u_{j, *}(x)\right||t|^{J}}{\Gamma(1+s j) j !(\sin (\delta))^{s J+1}} \int_{0}^{+\infty} u^{s J} e^{-u} d u \\
& =\sum_{j \geqslant 0} \frac{b^{j-J}\left|u_{j, *}(x)\right|}{\Gamma(1+s j) j !(\sin (\delta))^{s J+1}} \Gamma(1+s J)|t|^{J},
\end{aligned}
$$

where, according to the choice of $b$ (see the beginning of the proof), we have

$$
\sum_{j \geqslant 0} \frac{b^{j}\left|u_{j, *}(x)\right|}{\Gamma(1+s j) j !} \leqslant C \sum_{j \geqslant 0} \frac{\Gamma(1+(s+1) j)}{\Gamma(1+s j) j !}(K b)^{j}<+\infty .
$$

Consequently, we finally get

$$
\left|u(t, x)-\sum_{j=0}^{J-1} u_{j, *}(x) \frac{t^{j}}{j !}\right| \leqslant C^{\prime} K^{J} \Gamma(1+s J)|t|^{J},
$$

with $C^{\prime}=\frac{C}{\sin (\delta)} \sum_{j \geqslant 0} \frac{\Gamma(1+(s+1) j)}{\Gamma(1+s j) j !}(K b)^{j}$ and $K^{\prime}=\frac{1}{b(\sin (\delta))^{s}}$. The constants $C^{\prime}$ and $K^{\prime}$ depend on $\Sigma_{\delta}$ and on the choice of $b$, but are independant of $t$ and $x$. This achieves the proof.

\section{REFERENCES}

[1] W. Balser. Divergent solutions of the heat equation: on an article of Lutz, Miyake and Schäfke. Pacific J. Math., 188(1):53-63, 1999.

[2] W. Balser. Formal power series and linear systems of meromorphic ordinary differential equations. Universitext. Springer-Verlag, New-York, 2000.

[3] W. Balser. Multisummability of formal power series solutions of partial differential equations with constant coefficients. J. Differential Equations, 201(1):63-74, 2004.

[4] W. Balser and M. Loday-Richaud. Summability of solutions of the heat equation with inhomogeneous thermal conductivity in two variables. Adv. Dyn. Syst. Appl., 4(2):159-177, 2009 . 
[5] W. Balser and M. Miyake. Summability of formal solutions of certain partial differential equations. Acta Sci. Math. (Szeged), 65(3-4):543-551, 1999.

[6] W. Balser and M. Yoshino. Gevrey order of formal power series solutions of inhomogeneous partial differential equations with constant coefficients. Funkcial. Ekvac., 53:411-434, 2010.

[7] M. Canalis-Durand, J.-P. Ramis, R. Schäfke, and Y. Sibuya. Gevrey solutions of singularly perturbed differential equations. J. Reine Angew. Math., 518:95-129, 2000.

[8] O. Costin, H. Park, and Y. Takei. Borel summability of the heat equation with variable coefficients. J. Differential Equations, 252(4):3076-3092, 2012.

[9] M. Hibino. Borel summability of divergence solutions for singular first-order partial differential equations with variable coefficients. I \& II. J. Differential Equations, 227(2):499-563, 2006.

[10] M. Hibino. On the summability of divergent power series solutions for certain first-order linear PDEs. Opuscula Math., 35(5):595-624, 2015.

[11] K. Ichinobe. On $k$-summability of formal solutions for a class of partial differential operators with time dependent coefficients. J. Differential Equations, 257(8):3048-3070, 2014.

[12] A. Lastra and S. Malek. On parametric Gevrey asymptotics for some nonlinear initial value Cauchy problems. J. Differential Equations, 259:5220-5270, 2015.

[13] A. Lastra and S. Malek. On parametric multisummable formal solutions to some nonlinear initial value Cauchy problems. Adv. Differ. Equ., 2015:200, 2015.

[14] A. Lastra, S. Malek, and J. Sanz. On Gevrey solutions of threefold singular nonlinear partial differential equations. J. Differential Equations, 255:3205-3232, 2013.

[15] M. Loday-Richaud. Stokes phenomenon, multisummability and differential Galois groups. Ann. Inst. Fourier (Grenoble), 44(3):849-906, 1994.

[16] M. Loday-Richaud. Divergent Series, Summability and Resurgence II. Simple and Multiple Summability, volume 2154 of Lecture Notes in Math. Springer-Verlag, 2016.

[17] D. A. Lutz, M. Miyake, and R. Schäfke. On the borel summability of divergent solutions of the heat equation. Nagoya Math. J., 154:1-29, 1999.

[18] S. Malek. On the summability of formal solutions of linear partial differential equations. $J$. Dyn. Control Syst., 11(3):389-403, 2005.

[19] S. Malek. On singularly perturbed partial integro-differential equations with irregular singularity. J. Dyn. Control Syst., 13(3):419-449, 2007.

[20] S. Malek. On the summability of formal solutions of nonlinear partial differential equations with shrinkings. J. Dyn. Control Syst., 13(1):1-13, 2007.

[21] S. Malek. On the Stokes phenomenon for holomorphic solutions of integrodifferential equations with irregular singularity. J. Dyn. Control Syst., 14(3):371-408, 2008.

[22] S. Malek. On Gevrey functions solutions of partial differential equations with fuchsian and irregular singularities. J. Dyn. Control Syst., 15(2):277-305, 2009.

[23] S. Malek. On Gevrey asymptotic for some nonlinear integro-differential equations. J. Dyn. Control Syst., 16(3):377-406, 2010.

[24] S. Malek. On the summability of formal solutions for doubly singular nonlinear partial differential equations. J. Dyn. Control Syst., 18(1):45-82, 2012.

[25] B. Malgrange. Sommation des séries divergentes. Expo. Math., 13:163-222, 1995.

[26] B. Malgrange and J.-P. Ramis. Fonctions multisommables. Ann. Inst. Fourier (Grenoble), 42:353-368, 1992.

[27] S. Michalik. Summability of divergent solutions of the $n$-dimensional heat equation. J. Differential Equations, 229:353-366, 2006.

[28] S. Michalik. On the multisummability of divergent solutions of linear partial differential equations with constant coefficients. J. Differential Equations, 249:551-570, 2010.

[29] S. Michalik. Summability and fractional linear partial differential equations. J. Dyn. Control Syst., 16(4):557-584, 2010.

[30] S. Michalik. Multisummability of formal solutions of inhomogeneous linear partial differential equations with constant coefficients. J. Dyn. Control Syst., 18(1):103-133, 2012.

[31] S. Michalik. Corrigendum to "On the multisummability of divergent solutions of linear partial differential equations with constant coefficients" [j. differential equations 249 (3) (2010) 551570]. J. Differential Equations, 255:2400-2401, 2013.

[32] M. Miyake. Newton polygons and formal Gevrey indices in the Cauchy-Goursat-Fuchs type equations. J. Math. Soc. Japan, 43(2):305-330, 1991. 
[33] M. Miyake. Borel summability of divergent solutions of the Cauchy problem to nonKovaleskian equations. In Partial differential equations and their applications (Wuhan, 1999), pages 225-239. World Sci. Publ., River Edge, NJ, 1999.

[34] M. Miyake and Y. Hashimoto. Newton polygons and Gevrey indices for linear partial differential operators. Nagoya Math. J., 128:15-47, 1992.

[35] M. Nagumo. Über das Anfangswertproblem partieller Differentialgleichungen. Jap. J. Math., 18:41-47, 1942.

[36] S. Ouchi. Multisummability of formal solutions of some linear partial differential equations. J. Differential Equations, 185(2):513-549, 2002.

[37] S. Ouchi. Borel summability of formal solutions of some first order singular partial differential equations and normal forms of vector fields. J. Math. Soc. Japan, 57(2):415-460, 2005.

[38] M. E. Pliś and B. Ziemian. Borel resummation of formal solutions to nonlinear Laplace equations in 2 variables. Ann. Polon. Math., 67(1):31-41, 1997.

[39] J.-P. Ramis. Dévissage Gevrey. Astérisque, Soc. Math. France, Paris, 59-60:173-204, 1978.

[40] J.-P. Ramis. Les séries $k$-sommables et leurs applications. In Complex analysis, microlocal calculus and relativistic quantum theory (Proc. Internat. Colloq., Centre Phys., Les Houches, 1979), volume 126 of Lecture Notes in Phys., pages 178-199. Springer, Berlin, 1980.

[41] J.-P. Ramis. Théorèmes d'indices Gevrey pour les équations différentielles ordinaires. Mem. Amer. Math. Soc., 48:viii+95, 1984.

[42] P. Remy. Gevrey order and summability of formal series solutions of some classes of inhomogeneous linear partial differential equations with variable coefficients. J. Dyn. Control Syst., 22(4):693-711, 2016.

[43] P. Remy. Gevrey order and summability of formal series solutions of certain classes of inhomogeneous linear integro-differential equations with variable coefficients. J. Dyn. Control Syst., 23(4):853-878, 2017.

[44] H. Tahara and H. Yamazawa. Multisummability of formal solutions to the cauchy problem for some linear partial differential equations. J. Differential Equations, 255(10):3592-3637, 2013.

[45] A. Yonemura. Newton polygons and formal Gevrey classes. Publ. Res. Inst. Math. Sci., 26:197-204, 1990.

Laboratoire de Mathématiques de Versailles, Université de Versailles Saint-Quentin, 45 aVenue des Etats-Unis, 78035 Versailles cedex, France

Email address: pascal.remy@uvsq.fr ; pascal.remy.maths@gmail.com 